\title{
Schur function expansion for normal matrix model and associated discrete matrix models
}

\author{
A. Yu. Orlov* $\quad$ T. Shiota ${ }^{\dagger}$
}

\begin{abstract}
We consider Schur function expansion for the partition function of the model of normal matrices. We show that this expansion coincides with Takasaki expansion [5] for tau functions of Toda lattice hierarchy. We show that the partition function of the model of normal matrices is, at the same time, a partition function of certain discrete models, which can be solved by the method of orthogonal polynomials. We obtain discrete versions of various known matrix models: models of non-negative matrices, unitary matrices, normal matrices.
\end{abstract}

\section{Introduction}

The model of normal matrices (MNM) was introduced and studied in [1], 2] and [3]. A matrix $M$ is called normal, if it commutes with hermitian conjugated matrix:

$$
\left[M, M^{\dagger}\right]=0
$$

One can bring the matrix $M$ to its diagonal form via the unitary matrix $U: M=U Z U^{\dagger}$, where $Z=\operatorname{diag}\left(z_{1}, \ldots, z_{n}\right)$, with $z_{i}$ the eigenvalues of $M$. It is clear that $M^{\dagger}=U \bar{Z} U^{\dagger}$, where the diagonal matrix $\bar{Z}$ is the complex conjugate of $Z$.

The model of the normal matrices is defined by its partition function as follows:

$$
Z_{n}=C \int d \Omega(M) e^{\operatorname{Tr} V_{1}(M)+\operatorname{Tr} V_{2}\left(M^{\dagger}\right)+\operatorname{Tr} V\left(M, M^{\dagger}\right)},
$$

where $C$ is a normalization constant. The integration measure is defined as

$$
d \Omega(M)=d_{*} U|\Delta(z)|^{2} \prod_{i=1}^{n} d^{2} z_{i},
$$

where $d_{*} U$ is the Haar measure on the unitary group $U(n)$, and $\Delta(z)$ is the Vandermonde determinant:

$$
\Delta(z)=\operatorname{det}\left(z_{i}^{n-k}\right)_{i, k=1, \ldots, n}=\prod_{i<k}^{n}\left(z_{i}-z_{k}\right)
$$

For the case $n=0$, one sets $\Delta(z)=1$.

\footnotetext{
*Oceanology Institute, Nahimovskii Prospect 36, Moscow, Russia, email: orlovs@wave.sio.rssi.ru

${ }^{\dagger}$ Mathematics Department, Faculty of Science, Kyoto University, email: shiota@math.kyoto-u.ac.jp
} 
The potentials $V_{1}(M), V_{2}\left(M^{\dagger}\right)$ are defined by their Taylor series as follows:

$$
V_{1}(M):=\xi(\mathbf{t}, M):=\sum_{m=1}^{\infty} t_{m} M^{m}, \quad V_{2}\left(M^{\dagger}\right):=\xi\left(\mathbf{t}^{\prime}, M^{\dagger}\right)=\sum_{m=1}^{\infty} t_{m}^{\prime}\left(M^{\dagger}\right)^{m}
$$

where $\mathbf{t}=\left(t_{1}, t_{2}, \ldots\right), \mathbf{t}^{\prime}=\left(t_{1}^{\prime}, t_{2}^{\prime}, \ldots\right) ; t_{m}, t_{m}^{\prime}$ are called coupling constants. The interaction term $V$, a function of two variables, is not specified here. Integral (1.2) is supposed to be convergent; in the present paper, we consider it as its perturbation series in the coupling constants.

After the change of variables $M \rightarrow(U, Z)$ and the integration over $U(n)$, one obtains

$$
Z_{n}=Z_{n}\left(V ; \mathbf{t}, \mathbf{t}^{\prime}\right)=\int_{\mathbb{C}} \cdots \int_{\mathbb{C}}|\Delta(z)|^{2} \prod_{i=1}^{n} e^{V_{1}\left(z_{i}\right)+V_{2}\left(\bar{z}_{i}\right)} e^{V\left(z_{i}, \bar{z}_{i}\right)} d^{2} z_{i},
$$

where the integration is over the complex planes of eigenvalues $z_{i}$. Here we choose the normalization constant $C=C(n)$ in such a way that $C \cdot \operatorname{Vol}(U(n))=1$.

The model of normal matrices has applications in a description of quantum Hall droplets. As it was shown in [4, the particular case of the model, namely, the case when $V(z, \bar{z})=-|z|^{2}$ in its $n \rightarrow \infty$ limit, is related to the interface dynamics of water spot with a constant source surrounded by oil; in addition, it is related to certain old problems of complex analysis. The relation of these problems to Toda lattice equations [15] and its dispersionless analogue [8] was found and discussed in 4.

The integrable structure of the model of normal matrices was found and discussed in [3]. The coupling constants $\mathbf{t}, \mathbf{t}^{\prime}$ and the size of matrices $n$ were identified with the so-called Toda lattice higher times.

Remark 1 One can absorb both $V_{1}$ and $V_{2}$ into the term $V$. We prefer not to do so, since we are interested in the integrable structure of the model; we therefore keep the parameters $\mathbf{t}=\left(t_{1}, t_{2}, \ldots\right), \mathbf{t}^{\prime}=\left(t_{1}^{\prime}, t_{2}^{\prime}, \ldots\right)$ as the Toda lattice higher times, see below.

Remark 2 If the term $V$ enjoys axial symmetry

$$
V(z, \bar{z})=V_{\text {axi }}\left(|z|^{2}\right)
$$

then, for a certain choice of coupling constants, there exists a different integrable structure: partition function (1.2) is an infinite-soliton tau function of a "dual" TL hierarchy, higher times of the dual hierarchy being related to the moments of $e^{V_{\text {axi }}}$. See [19] about this and related topics.

Schur functions and partitions Polynomial functions in many variables, like the Schur functions, are parameterized by partitions. A partition is a sequence of non-negative integers in the non-increasing order:

$$
\lambda=\left(\lambda_{1}, \lambda_{2}, \ldots, \lambda_{r}, \ldots\right), \quad \lambda_{1} \geq \lambda_{2} \geq \ldots \geq \lambda_{r} \geq \ldots,
$$

where we identify $\left(\lambda_{1}, \ldots, \lambda_{n}\right)$ with $\left(\lambda_{1}, \ldots, \lambda_{n}, 0\right)$, and in the case of infinite sequence, assume $\lambda_{r}=0$ as $r \gg 0$. The $\lambda_{i}$ in (1.5) are called the parts of the $\lambda$. The number of nonzero parts is the length of $\lambda$, denoted by $\ell(\lambda)$. The sum of the parts is the weight of $\lambda$, denoted by $|\lambda|$. If $n=|\lambda|$, we say that the $\lambda$ is a partition of $n$. The partition of zero (where $\lambda_{1}=0$ ) is denoted by boldface $\mathbf{0}$, to distinguish it from number 0 . The set of all partitions, including $\mathbf{0}$, is denoted by $P$. 
The (Young) diagram of a partition $\lambda$ is defined as the set of points (or nodes) $(i, j) \in \mathbb{Z}^{2}$, such that $1 \leq j \leq \lambda_{i}$. Thus, it is a subset of a rectangular array with $\ell(\lambda)$ rows and $\lambda_{1}$ columns. We denote the diagram of $\lambda$ by the same symbol $\lambda$. For example,

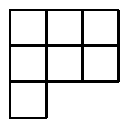

is the diagram of $(3,3,1)$. The weight of this partition is 7 , the length is equal to 3 .

The partition whose diagram is obtained by the transposition of the diagram $\lambda$ with respect to the main diagonal is called the conjugated partition and denoted by $\lambda^{t}$.

The product of hook lengths $H_{\lambda}$ is defined as

$$
H_{\lambda}=\prod_{i, j \in \lambda} h_{i j}, \quad h_{i j}=\lambda_{i}-i+\lambda_{j}^{t}-j+1
$$

where the product ranges over all nodes of the diagram of the partition $\lambda$.

Given number $q$, the so-called hook polynomial $H_{\lambda}(q)$ is defined as:

$$
H_{\lambda}(q)=\prod_{i, j \in \lambda}\left(1-q^{h_{i j}}\right), \quad h_{i j}=\lambda_{i}-i+\lambda_{j}^{t}-j+1
$$

In what follows, we also need notations:

$$
\begin{gathered}
n(\lambda):=\sum_{i=1}^{k}(i-1) \lambda_{i}, \\
(a)_{\lambda}:=(a)_{\lambda_{1}}(a-1)_{\lambda_{2}} \cdots(a-k+1)_{\lambda_{k}}, \quad(a)_{m}:=\frac{\Gamma(a+m)}{\Gamma(a)}, \\
\left(q^{a} ; q\right)_{\lambda}:=\left(q^{a} ; q\right)_{\lambda_{1}}\left(q^{a-1} ; q\right)_{\lambda_{2}} \cdots\left(q^{a-k+1} ; q\right)_{\lambda_{k}}, \quad\left(q^{a} ; q\right)_{m}:=\left(1-q^{a}\right) \cdots\left(1-q^{a+m-1}\right),
\end{gathered}
$$

where $k=\ell(\lambda)$. We set $(a)_{0}=1$ and $\left(q^{a} ; q\right)_{0}=1$.

We now consider a semi-infinite set of variables $\mathbf{t}=\left(t_{1}, t_{2}, t_{3}, \ldots\right)$. Given partition $\lambda$, the Schur function $s_{\lambda}(\mathbf{t})$ is defined by

$$
s_{\lambda}(\mathbf{t})=\operatorname{det}\left(h_{\lambda_{i}-i+j}(\mathbf{t})\right)_{1 \leq i, j \leq \ell(\lambda)}, \quad \text { where } \quad \sum_{k=0}^{\infty} z^{k} h_{k}(\mathbf{t})=\exp \sum_{m=1}^{\infty} z^{m} t_{m},
$$

and, for $k<0$, we put $h_{k}=0$. The $h_{k}(\mathbf{t})$ is called the elementary Schur function.

There is another definition of the Schur function; it is the following symmetric function in the different variables $x:=x^{(n)}:=\left(x_{1}, \ldots, x_{n}\right)$, where $n \geq \ell(\lambda)$ :

$$
\underline{s}_{\lambda}(x)=\frac{\operatorname{det}\left(x_{i}^{\lambda_{j}-j+n}\right)_{1 \leq i, j \leq n}}{\operatorname{det}\left(x_{i}^{n-j}\right)_{1 \leq i, j \leq n}} ;
$$

for the zero partition one puts $\underline{s}_{\mathbf{0}}(x)=1$. If

$$
\mathbf{t}=\mathbf{t}\left(x^{(n)}\right)=\left(t_{1}\left(x^{(n)}\right), t_{2}\left(x^{(n)}\right), \ldots\right), \quad t_{m}\left(x^{(n)}\right)=\frac{1}{m} \sum_{i=1}^{n} x_{i}^{m},
$$

then definitions (1.12) and (1.13) are equivalent 9$]$ :

$$
s_{\lambda}\left(\mathbf{t}\left(x^{(n)}\right)\right)=\underline{s}_{\lambda}\left(x^{(n)}\right) .
$$


Remark 3 From definition (1.12) it follows that $s_{\lambda}\left(\mathbf{t}\left(x^{(n)}\right)\right)=0$ if $\ell(\lambda)>n$.

The Schur functions $\underline{s}_{\lambda}\left(x_{1}, \ldots, x_{n}\right)$, where $\ell(\lambda) \leq n$, form a basis in the space of symmetric functions in $n$ variables. We use the underline in $\underline{s}_{\lambda}$ only to distinguish the two definitions. If an $n \times n$ matrix $X$ has eigenvalues $x_{1}, \ldots, x_{n}$, we may denote $\underline{s}_{\lambda}\left(x_{1}, \ldots, x_{n}\right)$ by $s_{\lambda}(X)$, without underline, since in this paper the Schur function with uppercase argument is used only in this sense.

Remark 4 If any of $x_{i}$ coincides with any of $\bar{y}_{i}$, say, $x_{k}=\bar{y}_{j}$, then we assume that the function $e^{V(z, \bar{z})}$ vanishes at $z=x_{k}^{-1}$ so that the integral (1.3) is convergent.

Soliton theory While the KP hierarchy of equations, [10, [12], [13, is the most popular example in the soliton theory, the two-dimensional Toda lattice (TL) is another important equation, first integrated in [14, and carefully studied in [15] in the framework of [12]:

$$
\partial_{t_{1}} \partial_{t_{1}^{\prime}} \varphi_{n}=e^{\varphi_{n+1}-\varphi_{n}}-e^{\varphi_{n}-\varphi_{n-1}}
$$

This equation gives rise to the TL hierarchy which contains derivatives with respect to the higher times $t_{1}, t_{2}, \ldots$ and $t_{1}^{\prime}, t_{2}^{\prime}, \ldots$ This equation was applied to the study of models of random Hermitian matrices (the so-called one- and two-matrix models) in [16], [17].

The key point of the soliton theory is the notion of tau function, introduced by Sato (for KP tau function; see [12]). The tau function, a sort of a potential which gives rise to the TL hierarchy, is a function of the two sequences of higher times, $t_{1}, t_{2}, \ldots$ and $t_{1}^{\prime}, t_{2}^{\prime}, \ldots$, and the discrete variable $n: \tau=\tau\left(n, \mathbf{t}, \mathbf{t}^{\prime}\right)$. More explicitly, we have [12], [15]:

$$
u=2 \partial_{t_{1}}^{2} \ln \tau\left(n, \mathbf{t}, \mathbf{t}^{\prime}\right), \quad \varphi_{n}\left(\mathbf{t}, \mathbf{t}^{\prime}\right)=\ln \frac{\tau\left(n+1, \mathbf{t}, \mathbf{t}^{\prime}\right)}{\tau\left(n, \mathbf{t}, \mathbf{t}^{\prime}\right)}
$$

Power series solutions The formal power series solutions of the TL hierarchy were described by Takasaki in the form of double series in the Schur functions over partitions [5] (see also [6], [7]):

$$
\tau\left(n, \mathbf{t}, \mathbf{t}^{\prime}\right)=\sum_{\lambda, \lambda^{\prime} \in P} g_{\lambda, \lambda^{\prime}}(n) s_{\lambda}(\mathbf{t}) s_{\lambda^{\prime}}\left(\mathbf{t}^{\prime}\right),
$$

where the coefficients $g_{\lambda, \lambda^{\prime}}$ can be presented as certain determinants or, alternatively, just solve special bilinear equations [12. We are interested in the semi-infinite Toda lattice, i.e., when the index $n$ runs over the set of nonnegative integers $\mathbb{Z}_{\geq 0}$. In the semi-infinite case, we have the restriction

$$
\ell(\lambda), \ell\left(\lambda^{\prime}\right) \leq n
$$

of the summation range on the right hand side of (1.16). By Theorem 1 of [5], in this case

$$
\begin{gathered}
g_{\lambda, \lambda^{\prime}}(n)=\operatorname{det}\left(G_{h_{i} h_{j}^{\prime}}\right)_{i, j=1, \ldots, n}, \\
h_{i}=\lambda_{i}-i+n \geq 0, \quad h_{i}^{\prime}=\lambda_{i}^{\prime}-i+n \geq 0,
\end{gathered}
$$

and semi-infinite matrix $G$ is related to the factorization problem $\Psi^{+}\left(\mathbf{t}, \mathbf{t}^{\prime}\right)=\Psi^{-}\left(\mathbf{t}, \mathbf{t}^{\prime}\right) G$, where the semi-infinite matrices $\Psi^{ \pm}$are Baker-Akhiezer functions [15].

If all but finitely many terms in (1.16) vanish, it gives a rational solution, in which case the formula may be best known. We are mainly interested in the case where (1.16) gives an infinite sum. 
Tau functions of hypergeometric type In the sequel, we shall also consider the diagonal case: $g_{\lambda, \lambda^{\prime}}(n)=0$ if $\lambda \neq \lambda^{\prime}$. In this case, generically (i.e., if $g_{\mathbf{0}, \mathbf{0}}(n) \neq 0(\forall n)$; this restriction is not essential) we have $g_{\lambda, \lambda^{\prime}}(n)=\delta_{\lambda, \lambda^{\prime}} r_{\lambda}(n) g_{\mathbf{0}, \mathbf{0}}(n)$, where the coefficients $r_{\lambda}(n)$ are given in the following way:

Consider a function $r=r(n)$ of a single variable $n \in \mathbb{Z}$. Given partition $\lambda$, we define

$$
r_{\lambda}(x)=\prod_{i, j \in \lambda} r(x+j-i), \quad r_{\mathbf{0}} \equiv 1 .
$$

Namely, we place $r(x+j-i)$ at each node $(i, j)$ of diagram $\lambda$, and multiply them over all nodes. The "content" $j-i$ of node $(i, j)$ vanishes on the main diagonal. For instance, if $\lambda=(3,3,1)$, then (see the diagram in (1.6) ) $r_{\lambda}(x)=r(x+2)(r(x+1))^{2}(r(x))^{2} r(x-1) r(x-2)$.

We define the tau function of hypergeometric type as

$$
\tau_{r}\left(n, \mathbf{t}, \mathbf{t}^{\prime}\right)=\sum_{\lambda} r_{\lambda}(n) s_{\lambda}(\mathbf{t}) s_{\lambda}\left(\mathbf{t}^{\prime}\right)
$$

which solves the TL equation (1.15), rewritten in the form

$$
\partial_{t_{1}} \partial_{t_{1}^{\prime}} \phi_{n}=r(n) e^{\phi_{n-1}-\phi_{n}}-r(n+1) e^{\phi_{n}-\phi_{n+1}}, \quad \varphi_{n}=-\phi_{n}+\xi_{n},
$$

where we set $r(k)=e^{\xi_{k}-\xi_{k-1}}$ and

$$
\phi_{n}\left(\mathbf{t}, \mathbf{t}^{\prime}\right)=-\ln \frac{\tau_{r}\left(n+1, \mathbf{t}, \mathbf{t}^{\prime}\right)}{\tau_{r}\left(n, \mathbf{t}, \mathbf{t}^{\prime}\right)}
$$

When $r(0)=0$, we obtain semi-infinite Toda lattice (1.18). In what follows, when we consider semi-infinite Toda tau function of the form $\tau_{r}$, we always assume $r(0)=0$.

A simplest example yields a useful and simple relation (bosonic (left hand side) and fermionic (right hand side) representations of the vacuum TL tau:

$$
\exp \sum_{m=1}^{\infty} m \gamma_{m} \gamma_{m}^{\prime}=\sum_{\lambda \in P} s_{\lambda}(\gamma) s_{\lambda}\left(\gamma^{\prime}\right)
$$

which is a generalized version of the Cauchy-Littlewood identity [9]. Here we use notations $\boldsymbol{\gamma}=\left(\gamma_{1}, \gamma_{2}, \ldots\right)$ and $\boldsymbol{\gamma}^{\prime}=\left(\gamma_{1}^{\prime}, \gamma_{2}^{\prime}, \ldots\right)$ in place of $\mathbf{t}$ and $\mathbf{t}^{\prime}$, for future purposes. (Since we did not find this identity in literature, we presented a proof in [23]).

\section{Discrete matrix models}

By discrete matrix model we mean models of random matrices, with eigenvalues lying on a lattice, the type of the lattice is different for different models and will be specified later in the section "Associated discrete models and discrete one-matrix models". We shall consider discrete counterparts of the following models, where integrals over eigenvalues are replaced by sums. Namely, we shall consider the following replacements.

Model of normal matrices:

$$
\int_{\mathbb{C}^{n}}|\Delta(z)|^{2} \prod_{i=1}^{n} e^{V_{1}\left(z_{i}\right)+V_{2}\left(\bar{z}_{i}\right)} e^{V\left(z_{i}, \bar{z}_{i}\right)} d^{2} z_{i} \rightarrow \sum \cdots \sum|\Delta(z)|^{2} \prod_{i=1}^{n} e^{V_{1}\left(z_{i}\right)+V_{2}\left(\bar{z}_{i}\right)} e^{V\left(z_{i}, \bar{z}_{i}\right)}=Z_{1}
$$


Model of two positive Hermitian matrices:

$$
\int_{\mathbb{R}_{+}^{2 n}} \Delta(x) \Delta(y) \prod_{i=1}^{n} e^{V_{1}\left(x_{i}\right)+V_{2}\left(y_{i}\right)} e^{V\left(x_{i}, y_{i}\right)} d x_{i} d y_{i} \rightarrow \sum \cdots \sum \Delta(x) \Delta(y) \prod_{i=1}^{n} e^{V_{1}\left(x_{i}\right)+V_{2}\left(y_{i}\right)} e^{V\left(x_{i}, y_{i}\right)}=Z_{2}
$$

Let us notice that the well-known standard two-matrix model is described by specific choice of the interaction term: $V(x, y)=x y$.

Model of positive Hermitian matrices:

$$
\int_{\mathbb{R}_{+}^{n}} \Delta(x)^{2} \prod_{i=1}^{n} e^{V\left(x_{i}\right)} d x_{i} \rightarrow \sum \cdots \sum \Delta(x)^{2} \prod_{i=1}^{n} e^{V\left(x_{i}\right)}=Z_{3}
$$

Model of two unitary matrices

$$
\oint \Delta(x) \Delta(y) \prod_{i=1}^{n} e^{V_{1}\left(x_{i}\right)+V_{2}\left(y_{i}\right)} e^{V\left(x_{i}, y_{i}\right)} \frac{d x_{i} d y_{i}}{x_{i} y_{i}} \rightarrow \sum \cdots \sum \Delta(x) \Delta(y) \prod_{i=1}^{n} e^{V_{1}\left(x_{i}\right)+V_{2}\left(y_{i}\right)} e^{V\left(x_{i}, y_{i}\right)}=Z_{4}
$$

where, for the standard case, one takes $V(x, y)=x^{-1} y^{-1}$.

Model of unitary matrices

$$
\oint|\Delta(z)|^{2} \prod_{i=1}^{n} e^{V_{1}\left(z_{i}\right)+V_{2}\left(\bar{z}_{i}\right)} e^{V\left(z_{i}, \bar{z}_{i}\right)} d^{2} z_{i} \rightarrow \sum \cdots \sum|\Delta(z)|^{2} \prod_{i=1}^{n} e^{V_{1}\left(z_{i}\right)+V_{2}\left(\bar{z}_{i}\right)} e^{V\left(z_{i}, \bar{z}_{i}\right)}=Z_{5}
$$

Kontsevich-like model:

$$
\int_{\mathbb{R}_{+}^{n}} \Delta(x) \prod_{i=1}^{n} e^{x_{i} y_{i}} e^{V\left(x_{i}\right)} d x_{i} \rightarrow \sum \cdots \sum \Delta(x) \prod_{i=1}^{n} e^{x_{i} y_{i}} e^{V\left(x_{i}\right)}=Z_{6}
$$

The multiply integrals in the left hand sides are integrals over the eigenvalues of the related matrix models. The cases of the generalized interaction term $V(x, y)$ in (2.2) and (2.4) should be considered separetely in order to be considered as integrals over matices. We do it in section "Two-matrix models with generalized interaction term" below.

What we are going to prove is that by choosing parameters $\mathbf{t}, \mathbf{t}^{\prime}$ in a special way we obtain

$$
Z_{n}\left(V ; \mathbf{t}, \mathbf{t}^{\prime}\right)=\text { patition function of discrete matrix models }, Z_{1}, \ldots, Z_{6}
$$

\section{Schur function expansion of the partition function}

In [22, [23], for the Gaussian interaction term $V(z, \bar{z})=-|z|^{2}$, the following perturbation series was obtained

$$
Z_{n}\left(V ; \mathbf{t}, \mathbf{t}^{\prime}\right)=n ! \sum_{\substack{\lambda \in P \\ \ell(\lambda) \leq n}}(n)_{\lambda} s_{\lambda}(\mathbf{t}) s_{\lambda}\left(\mathbf{t}^{\prime}\right),
$$

where $(n)_{\lambda}$ is defined in (1.10). At the present paper we generalize this result.

Given $V(z, \bar{z})$, let us introduce bi-moments $g_{k m}$, where $k, m=0,1,2, \ldots$, as

$$
g_{k m}=\int z^{k} \bar{z}^{m} e^{V(z, \bar{z})} d^{2} z,
$$

assuming the integral on the right hand side is well-defined. 
Theorem 1 The partition function of the model of the normal matrices (1.3) has the following perturbation series in the coupling constants $\mathbf{t}$ and $\mathbf{t}^{\prime}$ :

$$
Z_{n}\left(V ; \mathbf{t}, \mathbf{t}^{\prime}\right)=n ! \sum_{\substack{\lambda, \lambda^{\prime} \in P \\ \ell(\lambda), \ell\left(\lambda^{\prime}\right) \leq n}} g_{\lambda, \lambda^{\prime}}(n) s_{\lambda}(\mathbf{t}) s_{\lambda^{\prime}}\left(\mathbf{t}^{\prime}\right),
$$

where

$$
\begin{gathered}
g_{\lambda, \lambda^{\prime}}(n)=\operatorname{det}\left(g_{h_{i} h_{j}^{\prime}}\right)_{i, j=1, \ldots, n}, \\
h_{i}=\lambda_{i}-i+n \geq 0, \quad h_{i}^{\prime}=\lambda^{\prime}{ }_{i}-i+n \geq 0
\end{gathered}
$$

Proof. The proof repeats one of the proofs of (3.1), written down in [22]. Taking $\gamma_{m}=t_{m}$, $\gamma_{m}^{\prime}=(1 / m) \operatorname{Tr} M^{m}$, and applying (1.19) to the term $e^{\operatorname{Tr} V_{1}}$, and applying it again to the term $e^{\operatorname{Tr} V_{2}}$, with $\gamma_{m}=t_{m}^{\prime}, \gamma_{m}^{\prime}=(1 / m) \operatorname{Tr}\left(M^{\dagger}\right)^{m}$, we obtain

$$
\begin{aligned}
& Z_{n}\left(V ; \mathbf{t}, \mathbf{t}^{\prime}\right) \\
& =\sum_{\substack{\lambda, \lambda^{\prime} \in P \\
\ell(\lambda), \ell\left(\lambda^{\prime}\right) \leq n}} s_{\lambda}(\mathbf{t}) s_{\lambda^{\prime}}\left(\mathbf{t}^{\prime}\right) \int_{\mathbb{C}^{n}} \operatorname{det}\left(z_{k}^{h_{i}}\right)_{1 \leq k, i \leq n} \operatorname{det}\left(\bar{z}_{k} h^{\prime}\right)_{1 \leq k, i \leq n} \prod_{i=1}^{n} e^{V\left(z_{i}, \bar{z}_{i}\right)} d^{2} z_{i},
\end{aligned}
$$

where we used definition (1.13) to get the determinants in the integrands; these determinants are parameterized by the sets $\left\{h_{i} \mid i=1, \ldots, n\right\}$ and $\left\{h_{i}^{\prime} \mid i=1, \ldots, n\right\}$. The rest of the proof is quite similar to the calculation suggested by John Harnad for [22].

Given a pair of partitions (or, by (3.5), given a pair of sets $\left\{h_{i},\right\},\left\{h_{i}^{\prime}\right\}$ ), we develop each determinant as a sum of $n$ ! monomial terms: the first determinant yields the sum of monomials over all permutations $\sigma$ of numbers $1, \ldots, n: \sum_{\sigma \in S_{n}}(-)^{\operatorname{sign}(\sigma)} \prod_{i=1}^{n} z_{i}^{h_{\sigma(i)}}$, each monomial is parameterized by the set of non-negative numbers $\left\{h_{i}\right\}$ and by an element of the permutation group $\sigma$. Similarly, the second determinant yields $\sum_{\sigma}(-)^{\operatorname{sign}(\sigma)} \prod_{i} \bar{z}_{i(i)}^{h_{\sigma(i)}^{\prime}}$, each monomial is parameterized by the set $\left\{h_{i}^{\prime}\right\}$ and by $\sigma$. After integration, each pair of monomials produces the product of $n$ integrals of type (3.2). Notice the restriction $\ell(\lambda), \ell\left(\lambda^{\prime}\right) \leq n$, which follows from Remark 3 . Given a pair of sets $\left\{h_{i}\right\}$ and $\left\{h_{i}^{\prime}\right\}$, we gather all the terms into determinant (3.4) and obtain the Theorem.

Note that the very first term of (3.3) yields the formula presented in [3]

$$
Z_{n}(V ; 0,0)=n ! g_{\mathbf{0}, \mathbf{0}}=n ! \operatorname{det}\left(\int z^{n-i} \bar{z}^{n-j} e^{V(z, \bar{z})} d^{2} z\right)_{i, j=1, \ldots, n}
$$

Corollary 1 Using Theorem 1 of [5], we get another proof of the fact that the partition function of the model of normal matrices is a tau function of the (semi-infinite) TL hierarchy, [3], [4].

Inverse moment problem One can ask what kind of potential $V(z, \bar{z})$ of MNM (1.2) provides a given form of potentials in the discrete matrix models we have obtained. We need to present the following comment.

Remark 5 Since the moments $g_{m n}$ are the higher derivatives at the origin of the Fourier transform of $e^{V}$, and since every formal power series is the (formal) Taylor series at the origin of some $C^{\infty}$ function, one can, in principle, regard the moments $g_{m n}$ as independent variables. Having this fact in mind, in the future we may write each bi-moment $g_{m n}$ of (3.2) in a form analogous to $e^{V_{1}+V_{2}} e^{V}$ in (1.3):

$$
g_{k m}=e^{\tilde{V}_{1}(k)+\tilde{V}_{2}(m)} e^{\tilde{V}_{k m}},
$$

and assume certain kind of parameter dependence for $\tilde{V}_{1}(k)$ and $\tilde{V}_{2}(m)$. 
Note that, since the Taylor series of a $C^{\infty}$ function does not determine the function uniquely, the moments $\left\{g_{m n}\right\}_{m, n \geq 0}$ do not determine $e^{V}$ uniquely. Moreover, in popular function spaces like $L^{2}$ or Sobolev, the set of all $e^{V}$ 's whose bi-moments coincide with given $\left\{g_{m n}\right\}$ is not closed and may even be dense, so it is hard to give an explicit method or formula to reconstruct one.

Nevertheless, among those $e^{V}$ there is at most one which decays exponentially, i.e., $O\left(e^{-\varepsilon|z|}\right)$ as $|z| \rightarrow \infty$, since the Fourier transform of exponentially decreasing function is real analytic, and hence is determined uniquely by its Taylor series.

Parameter dependence of $\left\{g_{k m}\right\}$, like in (3.6), may often be introduced easily: Suppose, as a function of $r \in \mathbb{R}$ and $w=e^{i \theta} \in\{|w|=1\}, e^{V(z, \bar{z})}=e^{V\left(r w, r w^{-1}\right)}$ has analytic continuation in the $w$-variable to $\mathbb{C} \backslash\{0\}$, and the resulting function still is rapidly decreasing in $r$ for any $w \in \mathbb{C} \backslash\{0\}$. (This is slightly more general than the axial symmetric case. It includes, e.g., polynomials in $z$ and $\bar{z}$ with coefficients being rapidly decreasing functions in $|z|$.$) Let$ $e^{\tilde{V}_{k m}}:=\int_{\mathbb{C}} z^{k} \bar{z}^{m} e^{V(z, \bar{z})} d^{2} z$. Then for any $q_{1}, q_{2}>0$ we have

$$
q_{1}^{-1} q_{2}^{-1} \int_{\mathbb{C}} z^{k} \bar{z}^{m} e^{V\left(q_{1}^{-1} z, q_{2}^{-1} \bar{z}\right)} d^{2} z=q_{1}^{k} q_{2}^{m} e^{\tilde{V}_{k m}}
$$

hence, introducing two sets of parameters $\tilde{\mathbf{t}}=\left(\tilde{t}_{1}, \tilde{t}_{2}, \ldots\right)$ and $\tilde{\mathbf{t}}^{\prime}=\left(\tilde{t}_{1}^{\prime}, \tilde{t}_{2}^{\prime}, \ldots\right)$, and setting

$$
e^{V_{\tilde{\mathbf{t}}, \tilde{\mathbf{t}}^{\prime}}(z, \bar{z})}:=e^{\xi\left(\tilde{\mathbf{t}}, q_{1}^{D}\right)+\xi\left(\tilde{\mathbf{t}}^{\prime}, q_{2}^{\bar{D}}\right)} e^{V(z, \bar{z})}=\sum_{i, j \geq 0} h_{i}(\tilde{\mathbf{t}}) h_{j}\left(\tilde{\mathbf{t}}^{\prime}\right) q_{1}^{-i} q_{2}^{-j} e^{V\left(q_{1}^{-i} z, q_{2}^{-j} \bar{z}\right)},
$$

where $D:=-z \partial / \partial z-1$ and $\bar{D}:=-\bar{z} \partial / \partial \bar{z}-1$, at least as a formal power series in $\tilde{\mathbf{t}}$ and $\tilde{\mathbf{t}}^{\prime}$ we have

$$
\int_{\mathbb{C}} z^{k} \bar{z}^{m} e^{V_{\tilde{\mathbf{t}}, \tilde{\mathbf{t}}^{\prime}}(z, \bar{z})} d^{2} z=e^{\xi\left(\tilde{\mathbf{t}}, q_{1}^{k}\right)+\xi\left(\tilde{\mathbf{t}}^{\prime}, q_{2}^{m}\right)} e^{\tilde{V}_{k m}}
$$

A simple diagram summarizes what we get:

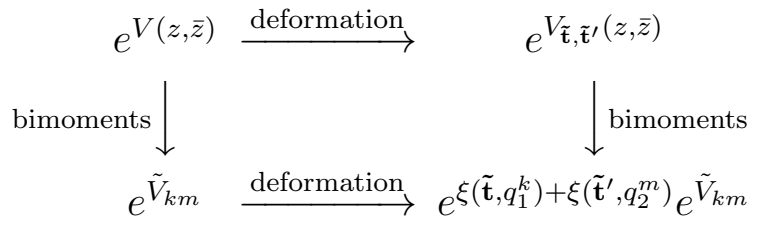

It is more illuminating to put $e^{\xi\left(\tilde{\mathbf{t}}, q_{1}^{D}\right)+\xi\left(\tilde{\mathbf{t}}^{\prime}, q_{2}^{\bar{D}}\right)} e^{V(z, \bar{z})}$ in place of $e^{V_{\tilde{\mathbf{t}}, \tilde{\mathbf{t}}^{\prime}}(z, \bar{z})}$ in this diagram, but the construction (3.7) may not work if $e^{V}$ is in more general form, or if the condition $q_{1}, q_{2}>0$ is not satisfied. Nevertheless, we can rely on less explicit argument in Remark 5 to assume the existence of $e^{V_{\tilde{\mathbf{t}}, \tilde{\mathbf{t}}^{\prime}}}$ subject to (3.8).

Axial-symmetric interaction term If we take axial-symmetric interaction term (1.4), $V(z, \bar{z})=V_{\text {axi }}\left(|z|^{2}\right)$, then $g_{k m}=\delta_{k m} g_{m m}$ is a diagonal matrix, which we parametrize by a set of numbers $\xi_{0}, \xi_{1}, \xi_{2}, \ldots$ as follows:

$$
g_{m m}=\pi \int_{0}^{+\infty} x^{m} e^{V_{\mathrm{axi}}(x)} d x=e^{\xi_{m}-\xi_{0}},
$$

so that

$$
g_{\lambda, \lambda^{\prime}}(n)=\delta_{\lambda, \lambda^{\prime}} \prod_{i=1}^{n} e^{\xi_{\lambda_{i}+n-i}-\xi_{n-i}}
$$

If both $\lambda, \lambda^{\prime}$ are zero partitions, we have $g_{\mathbf{0}, \mathbf{0}}=\prod_{m=0}^{n-1} g_{m m}$. Then we obtain

$$
Z_{n}\left(V_{\mathrm{axi}} ; \mathbf{t}, \mathbf{t}^{\prime}\right)=g_{\mathbf{0}, \mathbf{0}} n ! \sum_{\lambda \in P} e^{\sum_{i=1}^{\infty} \xi_{n+\lambda_{i}-i}-\xi_{n-i}} s_{\lambda}(\mathbf{t}) s_{\lambda}\left(\mathbf{t}^{\prime}\right)
$$


Let us notice that a number of matrix integrals (including the generalization of ItzyksonZuber, Gross-Witten and Kontsevich integrals, and various integrals over complex and rectangle matrices) may be written in form of series (3.10), where the variables $\xi$ are specialized via the choice of matrix model, see 20 .

In the following sections, we consider the right hand side of (3.3) and of (3.10) as models $Z_{1}, \ldots, Z_{6}$, where sums over numbers $h=\left(h_{1}, \ldots, h_{n}\right)$ and $h^{\prime}=\left(h_{1}^{\prime}, \ldots, h_{n}^{\prime}\right)$ play the role of sums over eigenvalues.

\section{Specialization of coupling constants. Associated dis- crete models and discrete one-matrix models}

Using the Schur function development (3.3), we shall show that there exist associated discrete models of random matrices, whose partition functions coincide with the partition function of the model of the normal matrices.

We introduce the following notations:

$$
\begin{gathered}
\mathbf{t}_{\infty}=(1,0,0,0, \ldots), \\
\mathbf{t}(a, 1)=\left(\frac{a}{1}, \frac{a}{2}, \frac{a}{3}, \ldots\right), \\
\mathbf{t}(\infty, q)=\left(t_{1}(\infty, q), t_{2}(\infty, q), \ldots\right), \quad t_{m}(\infty, q)=\frac{1}{m\left(1-q^{m}\right)}, \quad m=1,2, \ldots, \\
\mathbf{t}(a, q)=\left(t_{1}(a, q), t_{2}(a, q), \ldots\right), \quad t_{m}(a, q)=\frac{1-\left(q^{a}\right)^{m}}{m\left(1-q^{m}\right)}, \quad m=1,2, \ldots
\end{gathered}
$$

These choice of times was also used in [18 - 21.

Note that $\mathbf{t}(a, q)$ tends to $\mathbf{t}(\infty, q)$ (resp. $\mathbf{t}(a, 1))$ as $a \rightarrow \infty$ (resp. $q \rightarrow 1$ ). As for $\mathbf{t}_{\infty}$, if $f$ satisfies $f\left(c t_{1}, c^{2} t_{2}, c^{3} t_{3}, \ldots\right)=c^{d} f\left(t_{1}, t_{2}, t_{3}, \ldots\right)$ for some $d \in \mathbb{Z}$, we have $\hbar^{d} f(\mathbf{t}(\infty, q)) \rightarrow f\left(\mathbf{t}_{\infty}\right)$ as $\hbar:=\ln q \rightarrow 0$.

Lemma 1 For a partition $\lambda=\left(\lambda_{1}, \lambda_{2}, \ldots\right)$, let $h_{i}:=n+\lambda_{i}-i(1 \leq i \leq n)$, where $\left.n \geq \ell(\lambda)\right)$. Then

$$
\begin{gathered}
s_{\lambda}\left(\mathbf{t}_{\infty}\right)=\frac{1}{H_{\lambda}}=\frac{\Delta(h)}{\prod_{i=1}^{n} h_{i} !}, \\
s_{\lambda}(\mathbf{t}(a, 1))=\frac{(a)_{\lambda}}{H_{\lambda}}=\frac{\Delta(h)}{\prod_{i=1}^{n} h_{i} !} \prod_{i=1}^{n} \frac{\Gamma\left(a-n+h_{i}+1\right)}{\Gamma(a-i+1)}, \\
s_{\lambda}(\mathbf{t}(\infty, q))=\frac{q^{n(\lambda)}}{H_{\lambda}(q)}=\frac{\Delta\left(q^{h}\right)}{\prod_{i=1}^{n}(q ; q)_{h_{i}}}, \\
s_{\lambda}(\mathbf{t}(a, q))=\frac{q^{n(\lambda)}\left(q^{a} ; q\right)_{\lambda}}{H_{\lambda}(q)}=\frac{\Delta\left(q^{h}\right)}{\prod_{i=1}^{n}(q ; q)_{h_{i}}} \prod_{i=1}^{n}\left(q^{a-i+1} ; q\right)_{h_{i}-n+i}, \\
\Delta(h):=\prod_{i<j}^{n}\left(h_{i}-h_{j}\right), \quad \Delta\left(q^{h}\right):=\prod_{i<j}^{n}\left(q^{h_{i}}-q^{h_{j}}\right),
\end{gathered}
$$

where for $H_{\lambda}, H_{\lambda}(q), n(\lambda),(a)_{\lambda}$ and $\left(q^{a} ; q\right)_{\lambda}$ see respectively (1.7), (1.8), (1.9), (1.10) and (1.11). Note that those quantities 4.5)-4.8) are independent of the choice of $n \geq \ell(\lambda)$. 
Relations (4.5) and (4.6) can be found in [9] (some notations are different) for $n=\ell(\lambda)$, and we only need to add that if we have (4.5) and other formulas in the Lemma for the value $n=\ell(\lambda)$, then we have it for all $n>\ell(\lambda)$, as seen by a straightforward calculation. First equalities in the relations (4.7) and (4.8) can be easily obtained from well-known relations of 9]. The second equality in (4.8) follows from known formulae of [9].

Using this Lemma, we consider the Schur function development of the partition function of the model of the normal matrices (3.3). Choosing the higher times according to (4.1), to (4.2), to (4.3), and to (4.4), we obtain the following four models (A), (B), (C) and (D), respectively:

(A) Hermitian discrete matrix models $Z_{2}, Z_{3}$ with positive eigenvalues Consider the case

$$
\mathbf{t}=\left(t_{1}, 0,0, \ldots\right)=t_{1} \mathbf{t}_{\infty}, \quad \mathbf{t}^{\prime}=\left(t_{1}^{\prime}, 0,0, \ldots\right)=t_{1}^{\prime} \mathbf{t}_{\infty}
$$

for model (1.2), and use $s_{\lambda}\left(t \mathbf{t}_{\infty}\right)=t^{|\lambda|} s_{\lambda}\left(\mathbf{t}_{\infty}\right)$. By Theorem 1 we have

$$
Z_{n}\left(V ; \mathbf{t}, \mathbf{t}^{\prime}\right)=C \int d \Omega(M) e^{\operatorname{Tr} V\left(M, M^{\dagger}\right)+t_{1} \operatorname{Tr} M+t_{1}^{\prime} \operatorname{Tr} M^{\dagger}}=n ! \sum_{\substack{\lambda, \lambda^{\prime} \\ \ell(\lambda), \ell\left(\lambda^{\prime}\right) \leq n}} t_{1}^{|\lambda|}\left(t_{1}^{\prime}\right)^{\left|\lambda^{\prime}\right|} g_{\lambda \lambda^{\prime}}(n) s_{\lambda}\left(\mathbf{t}_{\infty}\right) s_{\lambda^{\prime}}\left(\mathbf{t}_{\infty}\right)
$$

Note that this integral is a kind of Fourier transform.

For a pair of partitions $\lambda, \lambda^{\prime}$, take any $n \in \mathbb{Z}$ such that $n \geq \ell(\lambda), \ell\left(\lambda^{\prime}\right)$, and let $h_{i}:=n+\lambda_{i}-i$, $h_{i}^{\prime}:=n+\lambda_{i}^{\prime}-i$, for $1 \leq i \leq n$. Then by using (4.5), the perturbation series for the partition function of the model of the normal matrices becomes

$$
\begin{aligned}
& Z_{n}\left(V ; \mathbf{t}_{\infty}, \mathbf{t}_{\infty}\right) \\
& =n ! g_{\mathbf{0}, \mathbf{0}} \sum_{h_{1}>\ldots>h_{n} \geq 0} \sum_{h_{1}^{\prime}>\ldots>h_{n}^{\prime} \geq 0} \Delta(h) \Delta\left(h^{\prime}\right) \prod_{i=1}^{n} e^{-\ln \Gamma\left(h_{i}+1\right)-\ln \Gamma\left(h_{i}^{\prime}+1\right)} \operatorname{det}\left(g_{h_{i} h_{j}^{\prime}}\right)_{i, j=1, \ldots, n} \\
& =\frac{g_{\mathbf{0}, \mathbf{0}}}{n !} \sum_{h, h^{\prime} \geq 0} \Delta(h) \Delta\left(h^{\prime}\right) \prod_{i=1}^{n} e^{-\ln \Gamma\left(h_{i}+1\right)-\ln \Gamma\left(h_{i}^{\prime}+1\right)} \operatorname{det}\left(g_{h_{i} h_{j}^{\prime}}\right)_{i, j=1, \ldots, n},
\end{aligned}
$$

where we got the factor $1 / n$ ! instead of $n$ !, as we switched from the summation over the cones $h_{1}>\cdots>h_{n} \geq 0, h_{1}^{\prime}>\cdots>h_{n}^{\prime} \geq 0$ to the summations over all non-negative integers $h_{1}, \ldots, h_{n}$ and $h_{1}^{\prime}, \ldots, h_{n}^{\prime}$, denoted by $\sum_{h \geq 0}$ and by $\sum_{h^{\prime} \geq 0}$ throughout the text. According to (3.2), the factor $\operatorname{det}\left(g_{h_{i} h_{j}^{\prime}}\right)_{i, j=1, \ldots, n}$ is defined by the interaction term $V$ in the model of the normal matrices and describes the interaction between the random matrices of the discrete model.

Using the skew-symmetry of $\Delta(h) \Delta\left(h^{\prime}\right)$, one can replace the determinant on the right hand side of (4.10) by the product of $n$ terms $g_{h_{i} h_{i}^{\prime}}, i=1, \ldots, n$, getting $n$ ! as a multiplier.

Recall that $|\lambda|=\sum_{i} h_{i}-\left(n^{2}-n\right) / 2$. Finely, we find that the perturbation series for the partition function of the model of the normal matrices is equal to the following partition function of a discrete model:

$$
Z_{n}\left(V ; \mathbf{t}, \mathbf{t}^{\prime}\right)=\left(t_{1} t_{1}^{\prime}\right)^{\frac{n-n^{2}}{2}} g_{\mathbf{0}, \mathbf{0}} \sum_{h, h^{\prime} \geq 0} \Delta(h) \Delta\left(h^{\prime}\right) \prod_{i=1}^{n} e^{-\ln \Gamma\left(h_{i}+1\right)-\ln \Gamma\left(h_{i}^{\prime}+1\right)+h_{i} \ln t_{1}+h_{i}^{\prime} \ln t_{1}^{\prime}+\ln g_{h_{i} h_{i}^{\prime}}},
$$

where each sum ranges over the $n$-tuples of all non-negative integers, $h_{1}, \ldots, h_{n}$ and $h_{1}^{\prime}, \ldots, h_{n}^{\prime}$, respectively; the times $\mathbf{t}, \mathbf{t}^{\prime}$ in the left hand side are chosen according to (4.9), and we remind that

$$
g_{k m}=\int_{\mathbb{C}} z^{k} \bar{z}^{m} e^{V(z, \bar{z})} d^{2} z, \quad \Delta(h)=\prod_{i<j}^{n}\left(h_{i}-h_{j}\right), \quad \Delta\left(h^{\prime}\right)=\prod_{i<j}^{n}\left(h_{i}^{\prime}-h_{j}^{\prime}\right)
$$


We notice that the first non-trivial term in sum (4.11), corresponding to $h_{i}=h_{i}^{\prime}=n-i$, is equal to one.

In case $g_{k m}=f(k m) e^{\tilde{V}_{1}(k)+\tilde{V}_{2}(m)}$ (see Remark 5) the sum (4.11) is a discrete analogue of the model of two Hermitian random matrices with non-negative eigenvalues with generalized interaction term $Z_{2}$, see (7.13) below. In case $f(k m)=e^{-k m}$ we obtain a discrete analogue of standard model of two Hermitian random matrices.

If we take axial-symmetric interaction term (3.10), we obtain the partition function of the discrete one-matrix model of non-negative matrices $Z_{3}$ :

$$
Z_{n}\left(V_{\mathrm{axi}} ; \mathbf{t}_{\infty}, \mathbf{t}_{\infty}\right)=\left(t_{1} t_{1}^{\prime}\right)^{\frac{n-n^{2}}{2}} g_{\mathbf{0}, \mathbf{0}} \sum_{h \geq 0} \Delta(h)^{2} \prod_{i=1}^{n} e^{-2 \ln h_{i} !+h_{i} \ln \left(t_{1} t_{1}^{\prime}\right)+\xi_{h_{i}}-\xi_{n-i}}
$$

(B) We obtain different versions of models $Z_{2}, Z_{3}$ if we choose

$$
\mathbf{t}=\left(x a, x^{2} a, x^{3} a, \ldots\right), \quad \mathbf{t}^{\prime}=\left(y a^{\prime}, y^{2} a^{\prime}, y^{3} a^{\prime}, \ldots\right)
$$

Then, (as done in [20]) one can see that (1.2) takes the form

$$
Z_{n}\left(V ; \mathbf{t}, \mathbf{t}^{\prime}\right)=C \int d \Omega(M) e^{\operatorname{Tr} V\left(M, M^{\dagger}\right)} \operatorname{det}\left(I_{n}-x M\right)^{-a} \operatorname{det}\left(I_{n}-y M^{\dagger}\right)^{-a^{\prime}}
$$

Using results of [9] and the definition of $h_{i}$, we find that, for $\mathbf{t}$ as in (4.13),

$$
s_{\lambda}(\mathbf{t})=\frac{x^{|\lambda|}}{H_{\lambda}} \prod_{i=1}^{n} \frac{\Gamma\left(a-n+h_{i}+1\right)}{\Gamma(a-i+1)}
$$

Now, for $\mathbf{t}, \mathbf{t}^{\prime}$ of form (4.13), we get a partition function with the potential terms slightly different of the similar terms in (4.11):

$Z_{n}\left(V ; \mathbf{t}, \mathbf{t}^{\prime}\right)=(x y)^{\frac{n-n^{2}}{2}} g_{\mathbf{0}, \mathbf{0}} \sum_{h, h^{\prime} \geq 0} \Delta(h) \Delta\left(h^{\prime}\right) \prod_{i=1}^{n} e^{\ln \frac{\Gamma\left(a-n+h_{i}+1\right)}{\Gamma(a-i+1) \Gamma\left(h_{i}+1\right)}+\ln \frac{\Gamma\left(a^{\prime}-n+h_{i}^{\prime}+1\right)}{\Gamma\left(a^{\prime}-i+1\right) \Gamma\left(h_{i}^{\prime}+1\right)}+h_{i} \ln x+h_{i}^{\prime} \ln y+\ln g_{h_{i} h_{i}^{\prime}}}$

For axial-symmetric case (1.4), we obtain the following partition function of the discrete model of random non-negative matrices:

$$
Z_{n}\left(V_{\text {axi }} ; \mathbf{t}, \mathbf{t}^{\prime}\right)=(x y)^{\frac{n-n^{2}}{2}} g_{\mathbf{0}, \mathbf{0}} \sum_{h \geq 0} \Delta(h)^{2} \prod_{i=1}^{n} e^{\ln \frac{\Gamma\left(a-n+h_{i}+1\right) \Gamma\left(a^{\prime}-n+h_{i}+1\right)}{\Gamma(a-i+1) \Gamma\left(a^{\prime}-i+1\right)}-2\left(h_{i} !\right)^{2}+h_{i} \ln (x y)+\xi_{h_{i}}-\xi_{n-i}},
$$

where variables $T_{k}$ are related to the axial-symmetric $V$ via (3.9).

Another example: Take the axial symmetric Gauss interaction term $V_{\text {axi }}=-\operatorname{Tr}\left(M M^{\dagger}\right)$. Then

$$
\begin{aligned}
Z_{n}\left(V_{\text {axi }} ; \mathbf{t}(a, 1), \mathbf{t}_{\infty}\right) & =C \int d \Omega(M) e^{-\operatorname{Tr}\left(M M^{\dagger}\right)+t_{1} \operatorname{Tr} M} \operatorname{det}\left(I_{n}-y M^{\dagger}\right)^{-a}=g_{\mathbf{0}, \mathbf{0}} \sum_{\lambda}\left(y t_{1}\right)^{|\lambda|} \frac{(n)_{\lambda}(a)_{\lambda}}{H_{\lambda}^{2}} \\
& =\left(y t_{1}\right)^{\frac{n-n^{2}}{2}} g_{\mathbf{0}, \mathbf{0}} \sum_{h \geq 0} \Delta(h)^{2} \prod_{k=1}^{n} k ! e^{\ln \frac{\Gamma\left(a-n+h_{k}+1\right)}{\Gamma(a-i+1)}-2 \ln h_{k} !+h_{k} \ln \left(y t_{1}\right)}
\end{aligned}
$$


(C) Discrete models related to unitary, $Z_{4}, Z_{5}$, and to normal matrices $Z_{1}$ Choosing the times as in (4.3), we obtain that the partition function of the MNM (1.2) takes a form

$$
Z_{n}\left(V ; \mathbf{t}, \mathbf{t}^{\prime}\right)=C \int e^{\operatorname{Tr} V\left(M, M^{\dagger}\right)} \frac{d \Omega(M)}{\operatorname{det}\left(x M ; q_{1}\right)_{\infty} \operatorname{det}\left(y M^{\dagger} ; q_{2}\right)_{\infty}},
$$

where we use the notation (1.11) for $m=\infty$, and where

$$
\mathbf{t}=\left(x \frac{1}{1-q_{1}}, \frac{x^{2}}{2} \frac{1}{1-q_{1}^{2}}, \frac{x^{3}}{3} \frac{1}{1-q_{1}^{3}}, \ldots\right), \quad \mathbf{t}^{\prime}=\left(y \frac{1}{1-q_{2}}, \frac{y^{2}}{2} \frac{1}{1-q_{2}^{2}}, \frac{y^{3}}{3} \frac{1}{1-q_{2}^{3}}, \ldots\right)
$$

Using the arguments similar to the previous cases, we obtain the following discrete model

$$
Z_{n}\left(V ; \mathbf{t}, \mathbf{t}^{\prime}\right)=(x y)^{\frac{n-n^{2}}{2}} g_{\mathbf{0}, \mathbf{0}} \sum_{h, h^{\prime} \geq 0} \Delta\left(q_{1}^{h}\right) \Delta\left(q_{2}^{h^{\prime}}\right) \prod_{i=1}^{n} e^{-\ln \left(q_{1} ; q_{1}\right)_{h_{i}}-\ln \left(q_{2} ; q_{2}\right)_{h_{i}^{\prime}}{ }^{\prime} h_{i} \ln x+h_{i}^{\prime} \ln y+\ln g_{h_{h} h_{i}^{\prime}}}
$$

Let us take $q_{1}=q_{2}=q$ and $|q|=1$ (then we set $q=e^{\sqrt{-1} \hbar}$ ).

In case $g_{k m}=f\left(q^{k} q^{m}\right) e^{\tilde{V}_{1}\left(q^{-k}\right)+\tilde{V}_{2}\left(q^{-m}\right)}$ (see Remark 5) the sum (4.11) is a discrete analogue of the model of two unitary random matrices with generalized interaction term $Z_{4}$, see (7.13) below. In case $f\left(q^{k} q^{m}\right)=e^{q^{k} q^{m}}$ we obtain a discrete analogue of standard model of two unitary random matrices.

For axial-symmetric interaction term $V$ (1.4) in case $q_{1}=\bar{q}_{2}=q$ and using parametrization (3.9), we obtain the discrete model one-matrix model:

$$
Z_{n}\left(V_{\text {axi }} ; \mathbf{t}, \mathbf{t}^{\prime}\right)=(x y)^{\frac{n-n^{2}}{2}} g_{\mathbf{0}, \mathbf{0}} \sum_{h \geq 0}\left|\Delta\left(q^{h}\right)\right|^{2} \prod_{i=1}^{n} e^{-2 \ln (q ; q)_{h_{i}}+h_{i} \ln (x y)+\xi_{h_{i}}-\xi_{n-i}},
$$

which, in the case where $|q|=1$, can be interpreted as model of unitary matrices $Z_{5}$, and in the case where $|q|<1$, gives a discrete version of the model of the normal random matrices $Z_{1}$, where instead of the integration over the complex plane, we have summation over the spiral $\left\{q^{h} \mid h=0,1, \ldots\right\}$.

(D) This case is rather close to the previous one. The choice of times as

$$
\mathbf{t}=\left(x \frac{1-q_{1}^{a}}{1-q_{1}}, \frac{x^{2}}{2} \frac{1-q_{1}^{2 a}}{1-q_{1}^{2}}, \frac{x^{3}}{3} \frac{1-q_{1}^{3 a}}{1-q_{1}^{3}}, \ldots\right), \quad \mathbf{t}^{\prime}=\left(y \frac{1-q_{2}^{a^{\prime}}}{1-q_{2}}, \frac{y^{2}}{2} \frac{1-q_{2}^{2 a^{\prime}}}{1-q_{2}^{2}}, \frac{y^{3}}{3} \frac{1-q_{2}^{3 a^{\prime}}}{1-q_{2}^{3}}, \ldots\right)
$$

in the MNM model (1.2) yields the integral

$$
Z_{n}\left(V ; \mathbf{t}, \mathbf{t}^{\prime}\right)=C \int d \Omega(M) e^{\operatorname{Tr} V\left(M, M^{\dagger}\right)} \operatorname{det} \frac{\left(x q_{1}^{a} M ; q_{1}\right)_{\infty}\left(y q_{2}^{a^{\prime}} M^{\dagger} ; q_{2}\right)_{\infty}}{\left(x M ; q_{1}\right)_{\infty}\left(y M^{\dagger} ; q_{2}\right)_{\infty}}
$$

This matrix integral have the perturbation series, described by the following discrete model:

$$
Z_{n}\left(V ; \mathbf{t}, \mathbf{t}^{\prime}\right)=(x y)^{\frac{n-n^{2}}{2}} g_{\mathbf{0}, \mathbf{0}} \sum_{h, h^{\prime} \geq 0} \Delta\left(q_{1}^{h}\right) \Delta\left(q_{2}^{h^{\prime}}\right) \prod_{i=1}^{n} e^{\ln \frac{\left(q_{1}^{a-i+1} ; q_{1}\right)_{h_{i}-n+1}\left(q_{2}^{a^{\prime}-i+1} ; q_{2}\right)_{h_{i}^{\prime}-n+1}}{\left(q_{1} ; q_{1} h_{i}\left(q_{2} ; q_{2}\right)_{h_{i}^{\prime}}\right.}+h_{i} \ln x+h_{i}^{\prime} \ln y+\ln g_{h_{i} h_{i}^{\prime}}}
$$

For axial-symmetric case (1.4), and for the case $q_{1}=\bar{q}_{2}=q$, we have the model

$$
Z_{n}\left(V_{\mathrm{axi}} ; \mathbf{t}, \mathbf{t}^{\prime}\right)=(x y)^{\frac{n-n^{2}}{2}} g_{\mathbf{0}, \mathbf{0}} \sum_{h \geq 0}\left(\Delta\left(q^{h}\right)\right)^{2} \prod_{i=1}^{n} e^{\ln \frac{\left(q^{a-i+1} ; q\right)_{h_{i}-n+1}\left(q^{a^{\prime}-i+1 ; q}\right)_{h_{i}-n+1}}{\left(q_{; q}\right)_{h_{i}}}+h_{i} \ln (x y)+\xi_{h_{i}}-\xi_{n-i}},
$$

where the right hand side in the case $|q|=1$ can be interpreted as a discrete one-matrix model $Z_{5}$, and for $q_{1,2}=\rho e^{ \pm \sqrt{-1} \hbar}$ it is a version of discrete MNM model $Z_{1}$. 
(E) Kontsevich-like discrete model $Z_{6}$. To get model (2.6) we consider the axial symmetric case.

First, we choose the variables $\mathbf{t}^{\prime}=\mathbf{t}^{\prime}\left(y^{(n)}\right)$. Then, we specialize $\mathbf{t}$ according to any of (4.1), (4.2), (4.3), or (4.4).

Let us consider only the case when $\mathbf{t}$ is specialized by (4.4) (which is the most general case among specializations (4.1), (4.2), (4.3), (4.4)). Then, using (3.10), using definition (1.13) of the Schur function, using (4.8) and using antisymmetry of the Vandermonde determinant, we can rewrite (3.3) in the form

$$
Z_{n}\left(V_{\mathrm{axi}}, \mathbf{t}(a, q), \mathbf{t}\left(y^{(n)}\right)\right) \Delta(y)=g_{\mathbf{0}, \mathbf{0}} n ! \cdot \sum_{h \geq 0} \Delta\left(q^{h}\right) \frac{\prod_{i=1}^{n}\left(q^{a-i+1} ; q\right)_{h_{i}-n+i}}{\prod_{i=1}^{n}(q ; q)_{h_{i}}} e^{\sum_{i=1}^{\infty}\left(\xi_{h_{i}}-\xi_{n-i}+h_{i} \ln y_{i}\right)}
$$

By changing $\log y_{i} \rightarrow y_{i}$ we obtain $Z_{6}$.

We mark that the relations between continues and discrete models of type, different of presented here, were considered in the papers [24, 25].

\section{Dual Schur functions. Associated discrete models and discrete matrix models}

In this section, we shall specify the sets of coupling constants $\mathbf{t}$ and $\mathbf{t}^{\prime}$ in (3.3) in a different way, via partitions, say, $\lambda^{*}$ and $\lambda^{\prime *}$. To do it, we consider the Schur function $\underline{s}_{\lambda}(x)$ in (1.13) as the function of a partition $\lambda$. We show that if $x_{i}=q^{h_{i}^{*}}$, where $h_{i}^{*}$ are integers, one can interchange the roles of $x$ and $\lambda$. Using this fact we differently relate series (3.3) to discrete analogs of partition functions of matrix models.

Given partitions $\lambda$ and $\lambda^{*}$, whose lengths do not exceed a given number $n$, we let

$$
h_{i}=\lambda_{i}-i+n, \quad h_{i}^{*}=\lambda_{i}^{*}-i+n,
$$

so that we have $h_{1}>h_{2}>h_{3}>\cdots>h_{n} \geq 0$, and $h_{1}^{*}>h_{2}^{*}>h_{3}^{*}>\cdots>h_{n}^{*} \geq 0$. Let

$$
x_{i}^{*}=q^{h_{i}}, \quad x_{i}=q^{h_{i}^{*}}, \quad i=1, \ldots, n,
$$

where $q$ is a given complex number.

By definition (1.13) of the Schur function, we obtain the following Lemma

Lemma 2 For any $q$, let $x=\left(x_{1}, \ldots, x_{n}\right)$ and $x^{*}=\left(x_{1}^{*}, \ldots, x_{n}^{*}\right)$ be as in (5.2). Then we have

$$
\Delta(x) \underline{s}_{\lambda}(x)=\Delta\left(x^{*}\right) \underline{s}_{\lambda^{*}}\left(x^{*}\right)
$$

Thus, given $\underline{s}_{\lambda}(x)$, with $x$ as described above, we obtain the dual Schur function $s_{\lambda^{*}}\left(x^{*}\right)$, where the roles of $h$ and $h^{*}$ are interchanged. Letting $q \rightarrow 1$ in (5.3), we get $\Delta\left(h^{*}\right) s_{\lambda}(\mathbf{t}(n, 1))=$ $\Delta(h) s_{\lambda^{*}}(\mathbf{t}(n, 1))$ in accordance with (4.8).

We remark that the case (4.3) results from (5.2) for $h_{i}^{*}=n-i$ and $n \rightarrow \infty$.

Given complex $q_{1}$ and $q_{2}$ and given partitions $\lambda^{*}$ and $\lambda^{\prime *}$, we now consider

$$
x_{i}=q_{1}^{h_{i}^{*}}, \quad y_{i}=q_{2}^{h_{i}^{*}},
$$


where

$$
h_{i}^{*}=\lambda_{i}^{*}-i+n \geq 0, \quad h_{i}^{\prime *}=\lambda_{i}^{\prime *}-i+n \geq 0,
$$

putting

$$
\begin{gathered}
x_{i}^{*}=q_{1}^{h_{i}}, \quad y_{i}^{*}=q_{2}^{h_{i}^{\prime}}, \\
h_{i}=\lambda_{i}-i+n \geq 0, \quad h_{i}^{\prime}=\lambda^{\prime}{ }_{i}-i+n \geq 0
\end{gathered}
$$

We choose $\mathbf{t}(x)=\left(t_{1}, t_{2}, \ldots\right), \mathbf{t}^{\prime}(y)=\left(t_{1}^{\prime}, t_{2}^{\prime}, \ldots\right)$ as

$$
t_{m}=\frac{1}{m} \sum_{i=1}^{n} q_{1}^{m h_{i}^{*}}, \quad t_{m}^{\prime}=\frac{1}{m} \sum_{i=1}^{n} q_{2}^{m h_{i}^{\prime *}}, \quad m=1,2, \ldots
$$

For the case when $x_{i}=q^{n-i}, i=1, \ldots, n$, that is $\lambda^{*}=0$, we shall write

$$
\mathbf{t}(n, q)=\left(t_{1}(n, q), t_{2}(n, q), \ldots\right), \quad t_{m}(n, q)=\frac{1}{m} \sum_{i=1}^{n} q^{m(n-i)}, \quad m=1,2, \ldots
$$

Then, using Lemma 1, we obtain for (3.3)

$$
Z_{n}\left(V ; \mathbf{t}(x), \mathbf{t}^{\prime}(y)\right) \Delta(x) \Delta(y)=n ! \sum_{h, h^{\prime}} \operatorname{det}\left(g_{h_{i} h_{j}^{\prime}}\right)_{i, j=1, \ldots, n} s_{\lambda^{*}}\left(q_{1}^{h}\right) s_{\lambda^{\prime *}}\left(q_{2}^{h^{\prime}}\right) \Delta\left(q_{1}^{h}\right) \Delta\left(q_{2}^{h^{\prime}}\right),
$$

where the sums range over the cones

$$
\infty>h_{1}>\cdots>h_{n} \geq 0, \quad \infty>h_{1}^{\prime}>\cdots>h_{n}^{\prime} \geq 0,
$$

For the choice of $\mathbf{t}(x)$ and $\mathbf{t}^{\prime}(y)$, given by partitions $\lambda^{*}$ and $\lambda^{\prime *}$ via (5.6) and (5.4), we shall use the notation

$$
Z_{n}\left(V ; \lambda^{*}, \lambda^{\prime *} ; q_{1}, q_{2}\right):=Z_{n}\left(V ; \mathbf{t}(x), \mathbf{t}^{\prime}(y)\right) \Delta(x) \Delta(y)
$$

where the right hand side is defined by (3.3). The case $\lambda^{*}=\lambda^{*}=0$ is related to (5.7).

Let us note that, in the case $n \rightarrow \infty$, the choice of the coupling constants in form (5.6) is rather general.

Using skew-symmetry properties of the factors, and changing the sum over partitions to the sum over all non-negative integers, we get

$$
Z_{n}\left(V ; \lambda^{*}, \lambda^{\prime *} ; q_{1}, q_{2}\right)=n ! g_{\mathbf{0}, \mathbf{0}} \sum_{h, h^{\prime} \geq 0} \frac{1}{(n !)^{2}} \Delta\left(q_{1}^{h}\right) \Delta\left(q_{2}^{h^{\prime}}\right) s_{\lambda^{*}}\left(q_{1}^{h}\right) s_{\lambda^{\prime *}}\left(q_{2}^{h^{\prime}}\right) \prod_{i=1}^{n}\left(g_{h_{i} h_{i}^{\prime}}\right)
$$

where we use notation (5.8), and where the sums range over all the $n$-tuples of non-negative integers $h_{1}, \ldots, h_{n}$ and $h_{1}^{\prime}, \ldots, h_{n}^{\prime}$. (We kept $1 /(n !)^{2}$ only to explain that the $(n !)^{2}$ in the denominator is obtained when we change the sum over cones to the sum over all non-negative integers).

Associated discrete model Let us introduce the following partition function

$$
Z_{n}^{\text {discr }}\left(\tilde{V} ; \tilde{\mathbf{t}}, \tilde{\mathbf{t}}^{\prime} ; q_{1}, q_{2}\right)=\frac{1}{n !} \sum_{h, h^{\prime} \geq 0} \Delta\left(q_{1}^{h}\right) \Delta\left(q_{2}^{h^{\prime}}\right) \prod_{i=1}^{n} e^{\xi\left(\tilde{\mathbf{t}}, q_{1}^{h_{i}}\right)+\xi\left(\tilde{\mathbf{t}}^{\prime}, q_{2}^{h_{i}^{\prime}}\right)} e^{\tilde{V}_{h_{i} h_{i}^{\prime}}}
$$

This sum can be viewed as a partition function of different models, which are discrete analogue of the models of random matrices, the choice of the model of random matrices depending on the specialization of the complex numbers $q_{1}$ and $q_{2}$. For instance, for $q_{1}=\rho e^{\sqrt{-1} \hbar}$ and $q_{2}=\rho e^{-\sqrt{-1} \hbar}$, where $\rho \neq 1$, sum (5.9) can be considered as a discrete version of (1.3).

Considering the perturbation series in coupling constants $\tilde{\mathbf{t}}, \tilde{\mathbf{t}}^{\prime}$ of (5.9) we can repeat the same trick we used to get Theorem 1. We obtain the series of type (3.3). 
Theorem 2 Let

$$
\tilde{g}_{k m}=A \sum_{h, h^{\prime} \geq 0} \prod_{i=1}^{n} q_{1}^{k h_{i}} q_{2}^{m h^{\prime} i} e^{\tilde{V}_{h_{i} h^{\prime}}}(\neq \infty), \quad k, m=0,1,2, \ldots,
$$

where the sum range over all non-negative numbers $h_{1}, \ldots, h_{n}$ and $h_{1}^{\prime}, \ldots, h_{n}^{\prime}$, and $q_{1}$ and $q_{2}$ are given complex numbers. The perturbation series in the coupling constants $\tilde{\mathbf{t}}$ and $\tilde{\mathbf{t}}^{\prime}$ for partition function of the model (5.9) can be presented as follows:

$$
Z_{n}^{\text {discr }}\left(\tilde{V} ; \tilde{\mathbf{t}}, \tilde{\mathbf{t}}^{\prime} ; q_{1}, q_{2}\right)=\sum_{\substack{\lambda, \lambda^{\prime} \in P \\ \ell(\lambda), \ell\left(\lambda^{\prime}\right) \leq n}} \tilde{g}_{\lambda, \lambda^{\prime}}(n) s_{\lambda}(\tilde{\mathbf{t}}) s_{\lambda^{\prime}}\left(\tilde{\mathbf{t}}^{\prime}\right),
$$

where the sums range over all non-negative numbers $h_{1}, \ldots, h_{n}$ and $h_{1}^{\prime}, \ldots, h_{n}^{\prime}$, and where

$$
\begin{gathered}
\tilde{g}_{\lambda, \lambda^{\prime}}(n)=\operatorname{det}\left(\tilde{g}_{\tilde{h}_{k} \tilde{h}_{m}^{\prime}}\right)_{k, m=1, \ldots, n}, \\
\tilde{h}_{i}=\lambda_{i}-i+n \geq 0, \quad \tilde{h}_{i}^{\prime}=\lambda^{\prime}{ }_{i}-i+n \geq 0
\end{gathered}
$$

This shows that sum (5.9) is a TL tau function, where $n, \tilde{\mathbf{t}}, \tilde{\mathbf{t}}^{\prime}$ play the role of higher times.

Remark 6 The sums (4.10), (5.9) can be studied by the method of (discrete) orthogonal polynomials (about the discrete version see, for instance, [30]), which links these sums with tau functions of integrable hierarchies, as done in [16] for the continuous case (namely, when we have integrals, instead of sums).

Consider the differential operator $s_{\lambda^{*}}(\tilde{\partial})$, which is evaluated as the Schur function (1.12), where each variable $t_{m}$ is replaced by the differential operator $\tilde{\partial}_{m}=(1 / m)\left(\partial / \partial \tilde{t}_{m}\right)$, and where the partition $\lambda^{*}$ is related to the variables $x$ by (5.5) and (5.4). Similarly, consider $s_{\lambda^{* *}}\left(\tilde{\partial}^{\prime}\right)$, which is the Schur function (1.12), where each $t_{m}$ is replaced by $\tilde{\partial}_{m}^{\prime}=(1 / m)\left(\partial / \partial \tilde{t}_{m}^{\prime}\right)$, and where the partition $\lambda^{\prime *}$ is related to the variables $y$ by (5.5) and by (5.4).

By Lemma 2, we obtain

Theorem 3 Let $g_{k m}=e^{\tilde{V}_{k m}}$. Suppose conditions (3.2) and (5.10) of Theorems 1 and 2 are satisfied. Let $a_{n}:=g_{\mathbf{0}, \mathbf{0}}(n) / \tilde{g}_{\mathbf{0}, \mathbf{0}}(n)$. In the notation (5.8), we have

$$
\begin{gathered}
Z_{n}\left(V ; 0,0 ; q_{1}, q_{2}\right)=\left.a_{n} Z_{n}^{\text {discr }}\left(\tilde{V} ; \tilde{\mathbf{t}}, \tilde{\mathbf{t}}^{\prime} ; q_{1}, q_{2}\right)\right|_{\tilde{\mathbf{t}}, \tilde{\mathbf{t}}^{\prime}=0}, \\
Z_{n}\left(V ; \lambda^{*}, \lambda^{\prime *} ; q_{1}, q_{2}\right)=\left.a_{n} s_{\lambda^{*}}(\tilde{\partial}) s_{\lambda^{* *}}\left(\tilde{\partial^{\prime}}\right) \cdot Z_{n}^{\text {discr }}\left(\tilde{V} ; \tilde{\mathbf{t}}, \tilde{\mathbf{t}}^{\prime} ; q_{1}, q_{2}\right)\right|_{\tilde{\mathbf{t}}, \tilde{\mathbf{t}}^{\prime}=0}
\end{gathered}
$$

If, moreover, the potential $V$ is deformed to $V_{\tilde{\mathbf{t}}, \tilde{\mathbf{t}}^{\prime}}$ subject to the diagram (3.8), then we can remove the restriction to $\tilde{\mathbf{t}}, \tilde{\mathbf{t}}^{\prime}=0$ in (5.11) and (5.12):

$$
\begin{gathered}
Z_{n}\left(V_{\tilde{\mathbf{t}}, \tilde{\mathbf{t}}^{\prime}} ; 0,0 ; q_{1}, q_{2}\right)=a_{n} Z_{n}^{\text {discr }}\left(\tilde{V} ; \tilde{\mathbf{t}}, \tilde{\mathbf{t}}^{\prime} ; q_{1}, q_{2}\right) \\
Z_{n}\left(V_{\tilde{\mathbf{t}}, \tilde{\mathbf{t}}} ; \lambda^{*}, \lambda^{\prime *} ; q_{1}, q_{2}\right)=a_{n} s_{\lambda^{*}}(\tilde{\partial}) s_{\lambda^{* *}}\left(\tilde{\partial^{\prime}}\right) \cdot Z_{n}^{\text {discr }}\left(\tilde{V} ; \tilde{\mathbf{t}}, \tilde{\mathbf{t}}^{\prime} ; q_{1}, q_{2}\right)
\end{gathered}
$$

We hope that the problem of averaging of the partition function (1.3) over initial data, namely, over $\mathbf{t}$ and $\mathbf{t}^{\prime}$, can be related to the problem of random partitions [32], $\lambda^{*}$ and $\lambda^{\prime *}$. 
Axial-symmetric case (1.4) In this case $h=h^{\prime}$. We denote $\tilde{V}\left(h_{i}\right):=\tilde{V}_{h_{i} h_{i}}$. We have

$$
Z_{n}^{\operatorname{discr}}\left(\tilde{V} ; \tilde{\mathbf{t}}, \tilde{\mathbf{t}}^{\prime} ; q_{1}, q_{2}\right)=\frac{\tilde{g}_{\mathbf{0}, \mathbf{0}}}{n !} \sum_{h \geq 0} \Delta\left(q_{1}^{h}\right) \Delta\left(q_{2}^{h}\right) \prod_{i=1}^{n} e^{\xi\left(\tilde{\mathbf{t}}, q_{1}^{h_{i}}\right)+\xi\left(\tilde{\mathbf{t}}^{\prime}, q_{2}^{h_{i}}\right)} e^{\tilde{V}\left(h_{i}\right)}
$$

where the sum ranges over all non-negative integer $h_{1}, \ldots, h_{n}$, and

$$
Z_{n}\left(V_{\mathrm{axi}} ; \lambda^{*}, \lambda^{\prime *} ; q_{1}, q_{2}\right)=\left.a_{n} s_{\lambda^{*}}(\tilde{\partial}) s_{\lambda^{\prime *}}\left(\tilde{\partial}^{\prime}\right) \cdot Z_{n}^{\mathrm{discr}}\left(\tilde{V} ; \tilde{\mathbf{t}}, \tilde{\mathbf{t}}^{\prime} ; q_{1}, q_{2}\right)\right|_{\tilde{\mathbf{t}}, \tilde{\mathbf{t}}^{\prime}=0},
$$

where $\mathbf{t}(x)$ and $\mathbf{t}^{\prime}(y)$ are described by the partitions $\lambda^{*}$ and $\lambda^{\prime *}$ as described by (5.6) and (5.5) . For $\lambda^{*}=\lambda^{\prime *}=0$, we have $s_{\lambda^{*}}(\tilde{\partial})=s_{\lambda^{* *}}\left(\tilde{\partial}^{\prime}\right)=1$, therefore, the partition functions of the continues and discrete models coincide.

We have different models for different $q_{1}$ and $q_{2}$ :

(A) If $q_{1}=\bar{q}_{2}=e^{\sqrt{-1} \hbar}$ and all $\tilde{V}_{m m}=0$, then, (5.15), where $\tilde{p}=\tilde{p}^{\prime}=0$, is a discrete version of the partition function of unitary one-matrix model [17], 21], that is

$$
\int_{U(n)} e^{\sum_{i} t_{i} \operatorname{Tr} U^{i}+\sum_{i} t_{i}^{\prime} \operatorname{Tr} U^{-i}} d_{*} U=\sum_{\lambda: \ell(\lambda) \leq n} s_{\lambda}(\mathbf{t}) s_{\lambda}\left(\mathbf{t}^{\prime}\right)=\tau_{r}\left(n, \mathbf{t}, \mathbf{t}^{\prime}\right)
$$

where we set $\mathbf{t}=\tilde{\mathbf{t}}, \mathbf{t}^{\prime}=\tilde{\mathbf{t}}^{\prime}$. In (5.17), the $r$ is the following step function: $r(k)=1$ if $k>0$, $r(k)=0$ if $k \leq 0$.

(B) Now, $\bar{q}_{1}=q=\rho e^{\sqrt{-1} \hbar}$ is a complex conjugate to $q_{2}$, and $|\rho|,|\hbar| \ll 1$. Let each $t_{m}^{\prime}=\bar{t}_{m}$ is a complex conjugate to $t_{m}$, that is, by (5.6), we have $h_{i}^{*}=h_{i}^{\prime *}$ for each $i$. We obtain

$$
Z_{n}\left(V_{\text {axi }} ; 0,0 ; q, \bar{q}\right)=\frac{a_{n}}{n !} \sum_{h \geq 0}\left|\Delta\left(q^{h}\right)\right|^{2} \prod_{i=1}^{n} e^{\tilde{V}\left(h_{i}\right)}
$$

which is a discrete analogue of the axial-symmetric model of the normal matrices (1.3) and (1.4), and

$$
Z_{n}\left(V_{\mathrm{axi}} ; \lambda^{*}, \lambda^{\prime *} ; q_{1}, q_{2}\right)=\left.\frac{a_{n}}{n !} s_{\lambda^{*}}(\tilde{\partial}) s_{\lambda^{\prime *}}\left(\tilde{\partial}^{\prime}\right) \cdot \sum_{h \geq 0}\left|\Delta\left(q^{h}\right)\right|^{2} \prod_{i=1}^{n} e^{\xi\left(\tilde{\mathbf{t}}, q^{h_{i}}\right)+\xi\left(\tilde{\mathbf{t}}^{\prime}, \bar{q}^{\left.h_{i}\right)}\right.} e^{\tilde{V}\left(h_{i}\right)}\right|_{\tilde{\mathbf{t}}, \tilde{\mathbf{t}}^{\prime}=0}
$$

\section{Discrete models as soliton solutions}

Here we consider the axial-symmetric case. In Frobenius notations (see $[9]), \lambda=(\alpha \mid \beta)$, we have

$$
e^{\sum_{i=1}^{n}\left(\xi_{h_{i}}-\xi_{n-i}\right)}=e^{\sum_{i=1}^{k}\left(\xi_{n+\alpha_{i}}-\xi_{n-\beta_{i}-1}\right)}
$$

and the series (3.10) may be restated as

$$
Z_{n}\left(V_{\mathrm{axi}} ; \mathbf{t}, \mathbf{t}^{\prime}\right)=n ! g_{\mathbf{0}, \mathbf{0}}\left(1+\sum_{k=1}^{\infty} \sum_{\substack{\alpha_{1}>\cdots>\alpha_{k} \geq 0 \\ n>\beta_{1}>\cdots>\beta_{k} \geq 0}}^{\infty} e^{\sum_{i=1}^{k}\left(\xi_{n+\alpha_{i}}-\xi_{n-\beta_{i}-1}\right)} s_{(\alpha \mid \beta)}(\mathbf{t}) s_{(\alpha \mid \beta)}\left(\mathbf{t}^{\prime}\right)\right)
$$

According to (19]), when we specialize $\mathbf{t}$ by (4.1)-(4.4), we obtain soliton tau functions of certain (dual) TL equation.

For instance, if we take $\mathbf{t}=\mathbf{t}_{\infty}$, we obtain

$$
\left(n ! g_{\mathbf{0}, \mathbf{0}}\right)^{-1} Z_{n}\left(V_{\mathrm{axi}} ; \mathbf{t}_{\infty}, \mathbf{t}^{\prime}\right)=
$$




$$
1+\sum_{k=1}^{\infty} \sum_{\substack{\alpha_{1}>\cdots>\alpha_{k} \geq 0 \\ n>\beta_{1}>\cdots>\beta_{k} \geq 0}}^{\infty} \frac{\prod_{i<j}^{k}\left(\alpha_{i}-\alpha_{j}\right)\left(\beta_{i}-\beta_{j}\right)}{\prod_{i, j=1}^{k}\left(\alpha_{i}+\beta_{j}+1\right)} \frac{s_{(\alpha \mid \beta)}\left(\mathbf{t}^{\prime}\right)}{\prod_{i=1}^{k} \alpha_{i} ! \prod_{i=1}^{k} \beta_{i} !} e^{\sum_{i=1}^{k}\left(\xi_{n+\alpha_{i}}-\xi_{n-\beta_{i}-1}\right)}
$$

Then, if we take the collection $\xi_{0}, \xi_{1}, \xi_{2}, \ldots$ by $\xi_{m}:=\xi(\tilde{\mathbf{t}}, m+a)-\xi\left(\tilde{\mathbf{t}}^{\prime},(m+a)^{-1}\right)=\sum_{k=1}^{\infty}((m+$ $\left.a)^{k} \tilde{t}_{k}-(m+a)^{-k} \tilde{t}_{k}^{\prime}\right)$, which, for any choice of $\mathbf{t}^{\prime}$, is a degenerate soliton tau function of a (dual) TL hierarchy, where the variables $\tilde{t}_{k}, \tilde{t}_{k}{ }_{k}$ play the role of higher times, see [19] for details.

Another example: if we take $\mathbf{t}=\mathbf{t}(\infty, q)$ and choose the collection $\xi_{0}, \xi_{1}, \xi_{2}, \ldots$ by $\xi_{m}=$ $\xi\left(\tilde{\mathbf{t}}, q^{m}\right)-\xi\left(\tilde{\mathbf{t}^{\prime}}, q^{-m}\right)$, then we obtain another soliton tau function of the dual TL equation:

$$
1+\sum_{k=1}^{\infty} \sum_{\substack{\alpha_{1}>\cdots>\alpha_{k} \geq 0 \\ n>\beta_{1}>\cdots>\beta_{k} \geq 0}}^{\infty} \frac{\prod_{i<j}^{k}\left(q^{\alpha_{i}+1}-q^{\alpha_{j}+1}\right)\left(q^{-\beta_{j}}-q^{-\beta_{i}}\right)}{\prod_{i, j=1}^{k}\left(q^{-\beta_{i}}-q^{\alpha_{j}+1}\right)} \frac{s_{(\alpha \mid \beta)}\left(\mathbf{t}^{\prime}\right)}{\prod_{i=1}^{k}(q ; q)_{\alpha_{i}} \prod_{i=1}^{k}(q ; q)_{\beta_{i}}} e^{\sum_{i=1}^{k}\left(\xi_{n+\alpha_{i}}-\xi_{n-\beta_{i}-1}\right)}
$$

The partition functions of our discrete matrix models (4.12), (4.14), (4.15), and (4.16) are also soliton tau functions. This fact is clear after re-writing sums using the Frobenius notations.

It will be interesting to compare these results with [28], where some models of random matrices where interpreted as a limit of infinite-soliton tau function in a different way.

\section{Two-matrix models with generalized interaction term}

(A) Development of determinants in the Schur functions Consider the power series

$$
f(z)=\sum_{n=0}^{\infty} e^{\xi_{n}-\xi_{0}} z^{n}
$$

Lemma 3 Let $x=\left(x_{1}, \ldots, n\right), y=\left(y_{1}, \ldots, y_{n}\right)$. Then

$$
c_{n} \frac{\operatorname{det}\left(f\left(x_{i} y_{k}\right)\right)_{i, k=1, \ldots, n}}{\Delta(x) \Delta(y)}=\sum_{\lambda \in P ; \ell(\lambda) \leq n} e^{\sum_{i=1}^{n}\left(\xi_{h_{i}}-\xi_{n-i}\right)} \underline{s}_{\lambda}(x) \underline{s}_{\lambda}(y)
$$

where $\Delta(x)=\prod_{1 \leq i<j \leq n}\left(x_{i}-x_{j}\right), c_{1}=1$, and $c_{n}=\prod_{i=0}^{n-1} e^{\xi_{0}-\xi_{i}}$ for $n>1$.

Putting $a_{n}:=e^{\xi_{n}-\xi_{0}}$ so that $e^{\sum_{i=1}^{n}\left(\xi_{h_{i}}-\xi_{n-i}\right)}=c_{n} \prod_{i=1}^{n} a_{h_{i}}$, defining $n \times \infty$ matrix $M$ by $M_{i j}=a_{j} x_{i}^{j}$ and $\infty \times n$ matrix $N$ by $N_{j k}=y_{k}^{j}$, and using (1.13), we observe that (7.2) reduces to the following well-known formula for the determinant of product of two matrices:

$$
\operatorname{det}(M N)=\sum_{h_{1}>h_{2}>\ldots>h_{n} \geq 1} \operatorname{det}\left(M_{1, \ldots, n}^{h_{n}, \ldots, h_{1}}\right) \operatorname{det}\left(N_{h_{n}, \ldots, h_{1}}^{1, \ldots, n}\right) .
$$

While this may be the simplest proof of the lemma, the following proof based on the known facts about tau functions is equally simple: First, we identify $f\left(x_{i} y_{j}\right)$ with the tau function

$$
\tau_{r}\left(1, x_{i}, y_{j}\right)=1+r(1) x_{i} y_{j}+r(1) r(2) x_{i}^{2} y_{j}^{2}+\cdots
$$

by $r(k)=e^{\xi_{k}-\xi_{k-1}}$. It may be shown by Wick's theorem (or the determinantal Fay identity [29, Theorem 4.2]) that

$$
\tau_{r}\left(n, \mathbf{t}(x), \mathbf{t}^{*}(y)\right)=c_{n} \frac{\operatorname{det}\left(\tau_{r}\left(1, x_{i}, y_{j}\right)\right)_{i, k=1}^{n}}{\Delta(x) \Delta(y)}
$$


where

$$
c_{1}=1, \quad c_{n}=\prod_{k=1}^{n-1} r(k)^{k-n}, \quad n>1
$$

It means that for any power series $f$ as in (7.1) we have

$$
c_{n} \frac{\operatorname{det}\left(f\left(x_{i} y_{k}\right)\right)_{i, k=1, \ldots, n}}{\Delta(x) \Delta(y)}=\tau_{r}\left(n, \mathbf{t}\left(x^{(n)}\right), \mathbf{t}^{\prime}\left(y^{(n)}\right)\right):=\sum_{\lambda} e^{\xi_{h_{i}}-\xi_{n-i}} s_{\lambda}\left(\mathbf{t}\left(x^{(n)}\right)\right) s_{\lambda}\left(\mathbf{t}^{\prime}\left(y^{(n)}\right)\right)
$$

(Here $c_{n}^{-1} \Delta(x) \Delta(y)$ is assumed to be non-vanishing; otherwise formula (7.4) should be modified.)

Remark 7 Choosing $r(k)=(k+n-M)^{-1} \prod_{i=1}^{p}\left(k+a_{i}\right) \prod_{i=1}^{s}\left(k+b_{i}\right)^{-1}$ for the tau function of hypergeometric type, we obtain the hypergeometric functions of two matrix arguments $X, Y$ [27], [18]:

$$
\begin{array}{r}
{ }_{p} \mathcal{F}_{s}\left(\begin{array}{l}
a_{1}+M, \ldots, a_{p}+M \\
b_{1}+M, \cdots, b_{s}+M
\end{array} \mid X, Y\right)=\tau_{r}\left(M, \mathbf{t}\left(x^{(n)}\right), \mathbf{t}^{*}\left(y^{(n)}\right)\right)= \\
\sum_{\substack{\lambda \\
\ell(\lambda) \leq n}} \frac{\prod_{k=1}^{p} s_{\lambda}\left(\mathbf{t}\left(a_{k}+M, 1\right)\right)}{\prod_{k=1}^{s} s_{\lambda}\left(\mathbf{t}\left(b_{k}+M, 1\right)\right)}\left(s_{\lambda}\left(\mathbf{t}_{\infty}\right)\right)^{s-p+1} \frac{s_{\lambda}(X) s_{\lambda}(Y)}{s_{\lambda}\left(I_{n}\right)}
\end{array}
$$

Here $x^{(n)}=\left(x_{1}, \ldots, x_{n}\right)$ and $y^{(n)}=\left(y_{1}, \ldots, y_{n}\right)$ are the eigenvalues of the matrices $X$ and $Y$, respectively.

Examples of ${ }_{p} \mathcal{F}_{s}:{ }_{0} \mathcal{F}_{0}\left(X, I_{n}\right)=e^{\operatorname{Tr} X},{ }_{1} \mathcal{F}_{0}\left(a \mid X, I_{n}\right)=\operatorname{det}\left(I_{n}-X\right)^{-a}$. Example of (17.4):

$$
{ }_{p} \mathcal{F}_{s}\left(\begin{array}{c}
a_{1}+n, \ldots, a_{p}+n \\
b_{1}+n, \cdots, b_{s}+n
\end{array} \mid X, Y\right)=\frac{c_{n}}{\Delta(x) \Delta(y)} \operatorname{det}\left({ }_{p} F_{s}\left(\begin{array}{c}
a_{1}+1, \ldots, a_{p}+1 \\
b_{1}+1, \cdots, b_{s}+1
\end{array} \mid x_{i} y_{j}\right)\right)_{i, j=1}^{n},
$$

which relates hypergeometric function of matrix arguments to hypergeometric functions of one variable.

(B) Angle integration of determinants We shall exploit the following formulae for integrations of Schur functions over the unitary group [9]. Let $d_{*} U$ be the normalized Haar measure on $U(n)$, and let $\delta_{\mu, \lambda}$ be the Kronecker symbol. By $I_{n}$ we shall denote $n$ by $n$ unit matrix. Let $A$ and $B$ be $n$ by $n$ matrices with the eigenvalues $a_{1}, \ldots, a_{n}$ and $b_{1}, \ldots, b_{n}$ respectively. (We are interested mainly in hermitian and unitary matrices, where eigenvalues are, respectively, real numbers or numbers on the unit circle). Then

$$
\int_{U(n)} s_{\lambda}\left(A U B U^{\dagger}\right) d_{*} U=\frac{s_{\lambda}(A) s_{\lambda}(B)}{s_{\lambda}\left(I_{n}\right)},
$$

and then consider the following model of two hermitian (or two unitary) random matrices

$$
J_{n}=\int e^{\operatorname{Tr} \tilde{V}_{1}\left(M_{1}\right)+\operatorname{Tr} \tilde{V}_{2}\left(M_{2}\right)} g\left(M_{1} M_{2}\right) d M_{1} d M_{2}
$$

where the measures $d M_{1}$ and $d M_{2}$ are defined in a standard way (see [33]), and where the interaction term $g$ is a hypergeometric tau function (see (1.17)) of the form

$$
g(X)=\tau_{r}\left(n, \mathbf{t}(X), \mathbf{t}\left(I_{n}\right)\right):=\sum_{\substack{\lambda \in P \\ \ell(\lambda) \leq n}} e^{\xi_{h_{i}}-\xi_{n-i}} s_{\lambda}(X) s_{\lambda}\left(I_{n}\right)
$$


where $r(k)=e^{\xi_{k}-\xi_{k-1}}$ is not fixed, and where

$$
\mathbf{t}(X)=\left(\frac{1}{1} \operatorname{Tr} X, \frac{1}{2} \operatorname{Tr} X^{2}, \frac{1}{3} \operatorname{Tr} X^{3}, \ldots\right)
$$

Then due to (7.8) it is possible to perform the angle integration over $U(n)$, where $M_{1}=$ $U_{1} A U_{1}^{\dagger}, M_{2}=U_{2} B U_{2}^{\dagger}, A=\operatorname{diag}\left(a_{1}, \ldots, a_{n}\right), B=\operatorname{diag}\left(b_{1}, \ldots, b_{n}\right)$.

$$
\int_{U(n)} g\left(M_{1} M_{2}\right) d_{*} U=\tau_{r}\left(n, \mathbf{t}\left(M_{1}\right), \mathbf{t}\left(M_{2}\right)\right)
$$

Finely we obtain the following integral over eigenvalues $a_{i}$ and $b_{i}$ :

$$
\begin{aligned}
J_{n} & =c_{n} \int \Delta(a)^{2} \Delta(b)^{2} \frac{\operatorname{det} f\left(a_{i} b_{k}\right)}{\Delta(a) \Delta(b)} \prod_{i=1}^{n} e^{\tilde{V}_{1}\left(a_{i}\right)+\tilde{V}_{2}\left(b_{i}\right)} d a_{i} d b_{i} \\
& =n ! c_{n} \int \Delta(a) \Delta(b) \prod_{i=1}^{n} f\left(a_{i} b_{i}\right) e^{\tilde{V}_{1}\left(a_{i}\right)+\tilde{V}_{2}\left(b_{i}\right)} d a_{i} d b_{i}
\end{aligned}
$$

\section{Conclusion}

Starting with the partition function of the model of normal matrices and using the duality relations for Schur functions, we get a Zoo of discrete models. In axial symmetric case these discrete models may be identified with soliton tau functions. The next step is to study the $n \rightarrow \infty$ limit of these models. Our results means that we should obtain a behavior common for groups of different models. Let us notice that discrete models $Z_{1}-Z_{5}$, we have obtained, can be studied by the method of orthogonal polynomials. It will be interesting to consider continuous vs. discrete models in the framework of different duality problems, see [34. These topis together with a generalization of all results to multi-matrix models we shall study in a more detailed paper.

\section{Acknowledgements}

The authors thank Oleg Zaboronsky and, most of all, John Harnad for helpful discussions. A.O. thanks O. Zaboronsky, J. Harnad, and J.J. Nimmo for the organization of his visits respectively to Warwick, Oxford and Glasgow Universities in May-June 2003, where this work was started. The work was supported by the Russian Foundation for Fundamental Researches (Grant No 0202-17382), the Program of Russian Academy of Science "Mathematical Methods in Nonlinear Dynamics", and a grant-in-aid for the scientific research of Japanese Ministry of Education and Science.

\section{References}

[1] Chau, L-L. and Yu, Y.: Unitary polynomials in normal matrix model and wave functions for the fractional quantum Hall effect, Phys. Lett. bf A167 p. 452 (1992)

[2] Chau, L-L. and Zaboronsky, O.: Normal matrix model, Toda lattice hierarchy, and the twodimensional electron gaz in the strong magnetic field, Proceedings in memory of professor Wolfgang Kroll, ed. J.P.Hsu et. al., World Scientific, Singapore, 1997 
[3] Chau, L-L. and Zaboronsky, O.: On the Structure of Correlation Functions in the Normal Matrix Models, Commun. Math. Phys. 196 (1998) 203-247; hep-th/9711091

[4] Mineev-Weinstein, M., Wiegmann, P. and Zabrodin, A.: Integrable structure of interface dynamics, Phys. Rev. Lett. 84 (2000) 5106-5109; nlin.SI/0001007

[5] Takasaki, K.: Initial value problem for the Toda lattice hierarchy, Adv. Stud. Pure Math. 4 (1984) 139-163

[6] Takebe, T.: Representation Theoretical Meaning of Initial Value Problem for the Toda Lattice Hierarchy I, LMP 21 (1991) 77-84

[7] Takebe, T.: Representation Theoretical Meaning of Initial Value Problem for the Toda Lattice Hierarchy II, Publ. RIMS, Kyoto Univ. 27 (1991), 491-503

[8] Takebe, T. and Takasaki, K.: Integrable Hierarchies and Dispersionless Limit, Rev. Math. Phys. 7 (1995) 743-808; hep-th/94050096

[9] Macdonald, I.G.: Symmetric Functions and Hall Polynomials, Second edition, Clarendon Press, Oxford, 1995

[10] Zakharov, V.E. and Shabat, A.B.: J. Funct. Anal. Appl. 8 (1974) 226

[11] Date, E., Jimbo, M., Kashiwara, M. and Miwa, T.: Transformation groups for soliton equations. In: Jimbo, M. and Miwa, T. (eds) Nonlinear integrable systems-classical theory and quantum theory pp. 39-120, World Scientific, 1983

[12] Jimbo, M. and Miwa, T.: Solitons and Infinite Dimensional Lie Algebras, Publ. RIMS Kyoto Univ. 19 (1983) 943-1001

[13] L. A. Dickey, Soliton Equations and Hamiltonian Systems, World Scientific, Singapore, 1991

[14] Mikhailov, A.V.: On the Integrability of two-dimensional Generalization of the Toda Lattice, Letters in Journal of Experimental and Theoretical Physics 30 (1979) 443-448

[15] Ueno, K. and Takasaki, K.: Adv. Stud. Pure Math. 4 (1984) 1-95

[16] Gerasimov, A., Marshakov, A., Mironov, A., Morozov, A., and Orlov, A.Yu.: Matrix Models of 2D Gravity and Toda Theory, Nuclear Physics B 357 (1991) 565-618

[17] Zabrodin, A., Kharchev, S., Mironov, A., Marshakov, A. and Orlov, A.: Matrix Models among Integrable Theories: Forced Hierarchies and Operator Formalism, Nuclear Physics $B \mathbf{3 6 6}$ (1991) 569-601

[18] A.Yu. Orlov and D.M. Scherbin "Multivariate hypergeometric functions as tau functions of Toda lattice and Kadomtsev-Petviashvili equation", Physica D 152-153 (2001) 51-56

[19] Orlov, A.Yu.: Rational solutions of KP hierarchy as multisoliton solutions of a dual KP (and TL) hierarchy submitted to Phys. Lett. A; Hypergeometric tau functions $\tau\left(\mathbf{t}, T, \mathbf{t}^{*}\right)$ as $\infty$-soliton tau function in $T$ variables; solv-int/0305001

[20] Orlov, A.Yu.: New Solvable Matrix Integrals, International Journal of Modern Physics A Vol. 19, Supplement (2004) 276-293; nlin. SI/0209063 
[21] A. Yu. Orlov, "New Solvable Matrix Models- $U(n)$ case" Proceedings of the workshop Nonlinear Physics: Theory and Experiment. II, Università di Lecce-Consortium Einstein, 27 June-6 July 2002, Gallipoli, Italy; editors: M.J. Ablowitz, M. Boiti, F. Pempinelli, B. Prinari; World Scientific New Jersey, London, Singapore, Hong Kong (2002) pp. 99-100

[22] Harnad, J. and Orlov, A.Yu.: Schur Function Expansions of Matrix Integrals, preprint CRM 2001

[23] Harnad, J. and Orlov, A.Yu.: Scalar products of symmetric functions and matrix integrals, nlin.SI/0211051. (To appear in proceedings of NEEDS2002, eds., A. Gonzales, World Scientific, 2002.) Theoretical and Mathematical Physics 137 No 3 (2003) 1676-1690

[24] Kostov, I.K., Staudacher, M. and Wynter, T.: Complex Matrix Models and Statistics of Branched Covering of 2D Surfaces, Commun. Math. Phys. 191 (1998) 283-298; hep-th/9703189

[25] Kostov, I.K.: Exact Solution of the Six-Vertex Model on a Random Lattice, Nucl. Phys. B 575 (2000) 513-534; hep-th/9911023

[26] Zakharov, V.E., Manakov, S.V., Novikov, S.P. and Pitaevsky, L.P. The Theory of Solitons. The Inverse Scattering Method, Moscow, Nauka, 1980

[27] Vilenkin, N.Ya. and Klimyk, A.U.: Representation of Lie Groups and Special Functions. Volume 3: Classical and Quantum Groups and Special Functions, Kluwer Academic Publishers, 1992

[28] Spiridonov, V. and Loutsenko, I.M.: Soliton Solutions of Integrable Hierarchies and Coulomb Plasmas, Journal of statistical physics 99 (2000) 751-767; cond-mat/9909308

[29] Adler, M. and van Moerbeke, P.: The spectrum of coupled random matrices, Annals of Math., 149, 921-976 (1999)

[30] Borodin, A. and Boyarchenko, D.: Distribution of the first particle in discrete orthogonal polynomial ensembles math-ph/0204001

[31] S. Kharchev, A. Marshakov and A. Mironov and A. Morozov, Int. J. Mod. Phys. A10 2015 (1995), "Generalized Kazakov-Migdal-Kontsevich Model: group theory aspects", hep-th/9312210; Nucl. Phys. B397 339 (1993); hep-th/9203043

[32] Borodin, A. and Olshanski, G.: Random partitions and Gamma kernel, math-ph/0305043

[33] Mehta, M. L., Random Matrices, 2nd edition (Academic, San Diego, 1991).

[34] Harnad, J. and Its, A.: Integrable Fredholm Operators and Dual Isomonodromic Deformations", Commun. Math. Phys. 226, 497-530 (2002); solv-int/9706002 